CERN-EP/99-132

16 September 1999

\title{
High-resolution tracking using large capillary bundles filled with liquid scintillator
}

P. Annis ${ }^{a}$, A. Bay $^{b}$, L. Benussi ${ }^{a}$, N. Bruski ${ }^{c}$, S. Buontempo ${ }^{d}$, C. Currat ${ }^{b}$, N. D'Ambrosio ${ }^{c}$, R. van Dantzig ${ }^{e}$, J. Dupraz ${ }^{f}$, A. Ereditato ${ }^{d}$, J.P. Fabre ${ }^{f}$, V. Fanti ${ }^{a}$, J. Feyt ${ }^{f}$, D. Frekers ${ }^{c}$, A. Frenkel ${ }^{g}$, F. Galeazzi ${ }^{g}$, F. Garufi ${ }^{d}$, J. Goldberg ${ }^{h}$, S.V. Golovkin ${ }^{i}$, A.M. Gorin ${ }^{i}$, G. Grégoire ${ }^{j}$, K. Harrison ${ }^{g}$, K. Hoepfner ${ }^{h, 1}$, K. Holtz ${ }^{c, 2}$, J. Konijn ${ }^{e}$, E.N. Kozarenko ${ }^{k}$, I.E. Kreslo ${ }^{g}$, A.E. Kushnirenko ${ }^{i}$, B. Liberti ${ }^{g}$, G. Martellotti ${ }^{g}$, A.M. Medvedkov ${ }^{i}$, L. Michel ${ }^{j}$, P. Migliozzi ${ }^{d}$, C. Mommaert ${ }^{a}$, M.R. Mondardini ${ }^{l}$, J. Panman ${ }^{f}$, G. Penso ${ }^{g}$, Y.P. Petukhov ${ }^{k}$, D. Rondeshagen ${ }^{c}$, W. P. Siegmund ${ }^{m, 3}$, V. Tyukov ${ }^{k}$, G. Van Beek ${ }^{a}$, V.G. Vasil'chenko ${ }^{i}$, P. Vilain ${ }^{a}$, J.L. Visschers ${ }^{e}$, G. Wilquet ${ }^{a}$, K. Winter ${ }^{l}$, T. Wolff ${ }^{c}$, H.J. Wörtche ${ }^{c}$, H. Wong ${ }^{f, 4}$, K.V. Zimyn ${ }^{i}$

\author{
a IIHE, ULB-VUB, Bruxelles, Belgium \\ ${ }^{b}$ Université de Lausanne, Lausanne, Switzerland \\ c Westfälische Wilhelms-Universität, Münster, Germany \\ ¿ Università "Federico II" and INFN, Napoli, Italy \\ e NIKHEF, Amsterdam, The Netherlands \\ ${ }^{f}$ CERN, Genève, Switzerland \\ g Università "La Sapienza" and INFN, Roma, Italy \\ h TECHNION, Haifa, Israel \\ iHEP, Protvino, Russia \\ ${ }^{j}$ Université Catholique de Louvain, Louvain-La-Neuve, Belgium \\ k JINR, Dubna, Russia \\ ${ }^{l}$ Humboldt-Universität, Berlin, Germany \\ $m$ Schott Fiber Optics, Inc., Southbridge, USA.
}

(Submitted to Nuclear Instruments $\&$ Methods A)

\footnotetext{
${ }^{1}$ Now at DESY, Hamburg, Germany.

${ }^{2}$ Now at Johannes Gutenberg-Universität, Mainz, Germany.

${ }^{3}$ Now at TaperVision, Inc., Pomfret, CT, USA.

${ }^{4}$ Now at Inst. of Physics, Academia Sinica, Taipei, Taiwan, Republic of China.
} 


\begin{abstract}
We have developed large high-resolution tracking detectors based on glass capillaries filled with organic liquid scintillator of high refractive index. These liquid-core scintillating optical fibres act simultaneously as detectors of charged particles and as image guides. Track images projected onto the readout end of a capillary bundle are visualized by an optoelectronic chain consisting of a set of image-intensifier tubes followed by a photosensitive CCD or by an EBCCD camera. Two prototype detectors, each composed of $\approx 10^{6}$ capillaries with $20-25 \mu \mathrm{m}$ diameter and $0.9-1.8 \mathrm{~m}$ length, have been tested, and a spatial resolution of the order of $20-40 \mu \mathrm{m}$ has been attained. A high scintillation efficiency and a large light-attenuation length, in excess of $3 \mathrm{~m}$, was achieved through special purification of the liquid scintillator. Along the tracks of minimum-ionizing particles, the hit densities obtained were $\sim 8$ hits $/ \mathrm{mm}$ at the readout window, and $\sim 3 \mathrm{hits} / \mathrm{mm}$ at $\sim 1 \mathrm{~m}$ away. The level of radiation resistance of the prototype detectors is at least an order of magnitude higher than that of other tracking devices of comparable performance.
\end{abstract}

PACS: 29.40.Gx; 29.40.Mc; 42.79.Ls; 85.60.Gz

Keywords: Tracking and position-sensitive detectors; Scintillation detectors; Scintillating fibres; Optoelectronics; Image intensifiers; CCD 


\section{Introduction}

Tracking devices with a spatial resolution in the range of microns are of great interest in high-energy physics as microvertex detectors for the study of interaction vertex topologies, for example decay patterns of extremely short-lived particles like $\tau$-leptons or charmed and beauty hadrons. Following some attempts to use optical fibres based on scintillating glass or plastic [1-13], glass capillaries filled with liquid scintillator (LS) have become increasingly attractive when considering the various experimental requirements. Over the past few years, many groups have contributed to the development of the capillary technique [14-26] for high-quality track imaging, making significant progress in many areas. Coherent arrays of thin glass capillaries (diameter of $20-30 \mu \mathrm{m}$ ) currently allow visualization of minimum-ionizing particle trajectories with several hits per $\mathrm{mm}$ of track length, and with a spatial resolution of the order of ten microns. The efficient light transmission of liquid-core fibres makes possible the construction of large-volume detectors. The real-time readout is performed by an optoelectronic chain, consisting of Image Intensifiers (II's) coupled either to a traditional photosensitive Charge-Coupled Device (CCD) or to a newly developed Electron-Bombarded CCD (EBCCD) camera. These components lie in the mainstream of the industrial interest for low-light-level imaging technology, and their quality and performance parameters are continuously improving. Further, the high level of parallelism provided by the optoelectronic readout makes large detectors cost effective.

In this paper we first give a short overview of our work on scintillating microfibres over the past ten years, and we compare the main features of glass and plastic fibres ${ }^{1}$ with those of capillaries filled with LS. We then report on our recent progress using the capillary technique: bundle manufacturing, bundle filling with LS, studies of light yield, light attenuation and spatial resolution with large capillary bundles. The readout methods and the data analyses are described. The tracking capabilities of the new devices have been tested in a pilot experiment, installed upstream of the CHORUS detector [27] in the CERN wide-band neutrino beam. Results from this pilot experiment are also presented.

Our work has been carried out in the framework of the CERN Research and Development project RD46 [28], the main goal of which has been to study the capabilities of capillary-based detectors for high-precision tracking and vertex reconstruction in highenergy physics experiments.

\section{From glass and plastic fibres to glass capillaries}

In the last ten years we have performed extensive studies of scintillating-microfibre detectors based on glass fibres, on plastic fibres, and on glass capillaries filled with LS.

The glass-fibre detector that we tested [5-7] consisted of a $4 \times 4 \times 40 \mathrm{~mm}^{3}$ bundle of parallel fibres. Each fibre had a $25 \times 25 \mu \mathrm{m}^{2}$ core of GS1, a glass doped with cerium oxide $\left(\mathrm{Ce}_{2} \mathrm{O}_{3}\right)$. The cladding of each fibre was made of clear glass, $2 \mu \mathrm{m}$ thick.

The plastic-fibre detectors $[6,7,10-12]$ consisted of cylindrical bundles of hexagonal fibres. The dimensions of the bundles and of each fibre were similar to those of the glass-fibre detector. The fibre cores were either SCSN-81T scintillator or polystyrene

\footnotetext{
${ }^{1}$ The studies of glass and plastic microfibre bundles were carried out by a part of the present authors, in the framework of the WA84 experiment at CERN.
} 
doped with 1-phenyl-3-mesityl-2-pyrazoline (PMP). In both cases, the fibre cladding was polymethyl methacrylate, $3 \mu \mathrm{m}$ thick.

The detectors based on glass capillaries filled with liquid scintillator are the last type we have studied. Results on this technique have been published both for bundles of small cross-section [15-20] and, more recently, for large-volume bundles [21-25]. The latter have transverse dimensions of 20-40 $\mathrm{mm}$ and lengths of $90-180 \mathrm{~cm}$, while the diameter of individual capillaries is in the range $20-30 \mu \mathrm{m}$. The capillaries are filled with newly developed liquid scintillators [26], based on 1-methylnaphthalene doped with a pyrazoline derivative.

In comparing the performance of glass fibres, plastic fibres and glass capillaries filled with LS we consider five main characteristics, discussed below and reported in Table 1.

\section{- Light yield}

The light yield is measured as the number of photons emerging at the readout end of the bundle when a minimum-ionizing particle crosses the bundle perpendicularly and close to the readout window. Taking into account the emission spectrum of the scintillator and the spectral sensitivity of the first photocathode of the readout chain, this characteristic is usually expressed in terms of the number of hits per unit track length, each hit corresponding to a photoelectron emitted by the photocathode of the first image intensifier of the readout chain. This quantity is evaluated by extrapolating to $d=0$ the hit density measured on tracks crossing the bundle at different distances $d$ from the readout end. In our early tests of a detector based on GS1 glass fibres, a hit density of $\sim 2.1 \mathrm{hits} / \mathrm{mm}$ was measured, whilst for a bundle of plastic fibres the corresponding value was $\sim 1.8 \mathrm{hits} / \mathrm{mm}[7]$. More recently, a hit density of $\sim 8$ hits $/ \mathrm{mm}$ has been obtained [21-23,25] for glass capillaries filled with the new LS [26]. The much higher hit density observed in this latter case is due partly to a better scintillation efficiency, and partly to the use of an improved readout chain. Measurements for $60 \mu \mathrm{m}$ plastic fibres by another group [13] have yielded hit densities that, at short distances, are consistent with those given here for the glass capillaries filled with LS.

\section{- Attenuation length of light in bundles}

The light output depends on the distance $(d)$ between a particle's crossing point and the readout end of the bundle. For bundles a few centimetres long, such as the bundles of glass and plastic fibres, this dependence is nearly exponential. The attenuation lengths measured [7] are $\sim 2 \mathrm{~cm}$ for GS1 glass fibres, $\sim 9 \mathrm{~cm}$ for SCSN$81 \mathrm{~T}$ plastic fibre and $\sim 31 \mathrm{~cm}$ for PMP-doped polystyrene fibres. For long $(1-2 \mathrm{~m})$ glass capillaries filled with LS, the dependence of the light output on $d$ can be approximately described by the sum of two exponentials. The different LS's tested have an attenuation length $\lambda(d)$ in the range $30-70 \mathrm{~cm}$ for smaller $d$ values, and have $\lambda \geq 300 \mathrm{~cm}$ when $d$ is large [21-23,25].

\section{- Decay time of the scintillation light}

For many scintillators the time dependence of the light emission is non-exponential, due to the presence of several components in the emission process. If slow components are present, the scintillator may be unsuitable for high-rate experiments. This is the case for GS1 glass, which, in addition to a main fast $(\sim 70 \mathrm{~ns})$ component, has a large tail up to $1 \mathrm{~ms}$ [29]. For plastic and liquid scintillators slow components are negligible, their decay times being of the order of a few nanoseconds $[7,30]$. 
Table 1: Characteristics of detectors based on scintillating microfibres with diameters of $20-30 \mu \mathrm{m}$. The hit density, which is strongly dependent on the sensitivity of the readout chain, has not been reported.

\begin{tabular}{lccc}
\hline & $\begin{array}{c}\text { glass fibres } \\
(\text { GS1) }\end{array}$ & $\begin{array}{c}\text { plastic fibres } \\
\text { (polystyrene with } \\
\text { SCSN-81T or PMP) }\end{array}$ & $\begin{array}{c}\text { glass capillaries } \\
\text { (filled with LS) }\end{array}$ \\
\hline attenuation length $(\mathrm{cm})$ & 2 & $9-31$ & $90-300$ \\
decay time & $0.07-350 \mu \mathrm{s}$ & $\sim 3 \mathrm{~ns}$ & $6-8 \mathrm{~ns}$ \\
optical crosstalk & medium & high & low \\
radiation hardness (Mrad) & $0.5-5$ & $0.2-2$ & $\sim 100$ \\
\hline
\end{tabular}

\section{- Optical crosstalk}

Light that, at a bundle's readout end, emerges from a fibre different from the fibre in which it was produced by a traversing particle contributes to the image noise. This crosstalk between fibres can be strongly reduced by surrounding each fibre with a light-absorbing material known as an Extra-Mural Absorber (EMA). For the fibre diameter we are considering, the EMA thickness cannot be much more than $\sim 1 \mu \mathrm{m}$, otherwise the packing fraction of the bundle (i.e. the ratio between the useful scintillating volume and the bundle's total volume) becomes too small. The problem is to find an EMA material that has good light absorption in a thickness of the order of $1 \mu \mathrm{m}$ and meets the mechanical, thermal and chemical properties needed for the bundle manufacturing process. This problem has been solved for glass fibres, but not for plastic fibres. For capillary bundles, the crosstalk is strongly reduced by filling the interstices between capillaries with black glass.

In addition to the optical crosstalk, image noise can come from the image-intensifier chain and from the CCD readout system [31].

\section{- Radiation hardness}

The radiation hardness of the scintillating fibres is an important characteristic when the tracking devices have to operate in a high-activity environment. The absorbed dose can produce both a lower scintillation efficiency and increased light attenuation along the fibres. The overall effect on light output depends on the radiation hardness of the scintillator and on the length of the bundles. For the glass and plastic fibres considered here, the light yield is significantly reduced [32-36] for an absorbed dose of the order of a few Mrad. For the large capillary bundles filled with the new LS, the radiation hardness is much higher. Depending on the particular LS used, a dose of the order of $\sim 100 \mathrm{Mrad}$ can be absorbed with only a limited reduction in light yield [37]. 


\section{Manufacture of capillary bundles}

The manufacture of a large capillary bundle involves the solution of many technical problems, and only a few commercial companies ${ }^{2}$ are currently able to build capillarybased detectors. In the following we refer to two large capillary bundles produced by Schott, each bundle containing $\sim(0.5-1.4) \times 10^{6}$ capillaries with an inner diameter of $20-25 \mu \mathrm{m}$.

The choice of the type of glass for bundle manufacture is based on several requirements. First, it is necessary to have a low refractive index, so as to achieve the highest numerical aperture for the liquid-core fibres. Secondly, to reduce losses due to non-perfect total reflection of light, the inner surfaces of glass capillaries must be smooth. Finally, good chemical stability is needed. All requirements are met by the Schott Technical Glass Type 8250 , a borosilicate glass normally used for electron-tube envelopes. It has a refractive index of 1.49, an expansion coefficient of $5.0 \times 10^{-6} \mathrm{~K}^{-1}$, a density of $2.28 \mathrm{~g} / \mathrm{cm}^{3}$, a softening point of $720^{\circ} \mathrm{C}$, and class-3 chemical stability.

The two large bundles that we have tested have been manufactured with different processes. For the first bundle, a traditional procedure was adopted. Beginning with a preform consisting of a single glass tube, a multichannel array is obtained through a series of drawing steps alternating with assembly steps. Specifically, the manufacturing process commenced with an 8250 -glass tube of $43 \mathrm{~mm}$ outer diameter being drawn to a diameter of $1.84 \mathrm{~mm}$. These monocapillaries were stacked to form an hexagonal array of 547 pieces and were placed inside a tube of 8250 glass. Black glass rods were inserted in the interstices between the monocapillaries to act as EMA. The assembly was then drawn to give an hexagonal "multi" measuring $1.7 \mathrm{~mm}$ between opposite flat sides. Finally, 940 multies were gathered in a close-packed pattern, and were inserted into a square glass tube, constituting the outer part of the bundle. This assembly, a "multi-multi", was drawn to the desired final dimensions. In Fig. 1 we show a schematic view of the tower used for this last drawing step.

The bundle obtained with this manufacturing process (hereafter referred to as the "square bundle"), was drawn to a final cross-section of $2 \times 2 \mathrm{~cm}^{2}$ along the first $165 \mathrm{~cm}$ of its length, while in the last $20 \mathrm{~cm}$, near the bundle's readout end, the cross-section increased smoothly from $2 \times 2 \mathrm{~cm}^{2}$ to $3.4 \times 3.4 \mathrm{~cm}^{2}$. In the bundle's narrower part, which corresponded to the particle-detector proper, the multies (Fig. 2) had a transverse dimension of $\sim 700 \mu \mathrm{m}$, measured between opposite flat sides, while the inner and outer diameters of the capillaries were respectively $\sim 20 \mu \mathrm{m}$ and $25 \mu \mathrm{m}$. The bundle's tapered readout end acted as an image guide, and magnified the detected track images by a factor of 1.7. This magnification reduced the effect of the image-intensifier chain on the overall spatial resolution.

The packing fraction of a bundle obtained with this first technique is about $55 \%$. It is limited by a closing-up of capillaries that occurs in the final drawing step, and which is due to the surface tension of the glass.

In order to obtain a higher packing fraction, the second bundle that we have tested was manufactured with a new procedure. The first two steps of the procedure were similar to the corresponding steps of the traditional procedure except that each multi was drawn to the final bundle length. The ends of each multi were then quickly sealed using a flame, taking care not to heat the gas inside the multies. The multies were stacked

\footnotetext{
${ }^{2}$ We have tested different capillary bundles produced by three companies: Schott Fiber Optics Inc., Southbridge, MA 01550-1960, USA; TaperVision Inc., Pomfret, CT 06258, USA; Geosphaera Research Centre, Moscow 117261, Russia.
} 
in an hexagonal glass tube, which eventually formed the outer part of the bundle. The resulting assembly was inserted in a stainless-steel tube, and the remaining space inside this tube was filled with sand. The entire system was placed in an horizontal oven, and was heated for several hours following a controlled thermal cycle. During this last step, air inside the capillaries expanded and forced the multies against one another, so reducing the dead spaces and securing the multies in the outer glass tube. With this "expansion method" we obtained a bundle (hereafter referred to as the "hexagonal bundle") that was $\sim 90 \mathrm{~cm}$ long, and had an hexagonal cross-section with a transverse dimension of $\sim 40 \mathrm{~mm}$, measured between opposite flat sides. The inner and outer diameters of the capillaries were respectively $\sim 25 \mu \mathrm{m}$ and $30 \mu \mathrm{m}$, and the packing fraction was $\sim 67 \%^{3}$, significantly larger than that of the first bundle.

In Fig. 3 we show photographs taken through a microscope of capillary arrays manufactured with the two abovementioned procedures.

Once a bundle was fully assembled, its ends were cut using a fine-grain diamond saw, under a flux of cooling liquid. To prevent small glass particles and cooling liquid entering into the capillaries during the cutting, a nitrogen flux was blown into the bundle through the opposite end. This cutting stage produced a planar surface suitable for coupling the bundles to the first image intensifier of the optoelectronic readout chain.

The bundles obtained with the two manufacturing methods had transverse widths that varied by $\sim \pm 5 \%$ along the bundle length. In Fig. 4 we show the behaviour of this width as a function of the distance from the readout end, for the narrow part of the square bundle and for the hexagonal bundle.

In high-energy physics applications, a relevant characteristic of a particle detector is its radiation length. For the quoted packing fraction, and for a LS based on 1-methylnaphthalene [26], the radiation lengths of the square bundle and of the hexagonal bundle were respectively $19 \mathrm{~cm}$ and $24 \mathrm{~cm}$.

\section{Liquid scintillators}

In the past few years, many new LS's have been specially developed [26] with a view to their use in tracking detectors based on glass capillaries. The best of them [38] are based on 1-methylnaphthalene (1MN) or isopropylnaphthalene (IPN) doped with pyrazoline derivatives such as ${ }^{4} \mathrm{R} 6, \mathrm{R} 39, \mathrm{R} 45$ or $3 \mathrm{M}-15$.

The principal characteristics of these LS's, and the bundle filling procedure, are discussed below.

\section{- Emission spectrum and light yield}

For the scintillators named, the emission spectra peak in the green region $(490-500$ $\mathrm{nm}$ ), as in the example of Fig. 5, and the scintillation efficiencies are comparable with, or better than, those of standard plastic scintillators. The light yield depends on the concentration, $C$, of the dye in the solvent. Following an initial increase in light yield with $C$, a maximum is reached around $C=2-3 \mathrm{~g} / 1$, then at higher concentrations there is a plateau or a slight decrease [26]. As an example, Fig. 6 shows the scintillation efficiency of $1 \mathrm{MN}$ doped with R6 as a function of the dye concentration. A similar behaviour has been observed [26] for all of the LS's based on $1 \mathrm{MN}$ or IPN.

\footnotetext{
${ }^{3}$ A packing fraction of $\sim 80 \%$ was achieved for a small bundle manufactured by Geosphaera.

${ }^{4} \mathrm{R} 6, \mathrm{R} 39, \mathrm{R} 45$ and $3 \mathrm{M}-15$ are trademarks of the Geosphaera Research Centre.
} 


\section{- Local light emission}

Most of the light emission occurs within a few microns of the track of an ionizing particle, i.e. within a fibre that the particle crosses. This feature is important in allowing advantage to be taken of the potentially high spatial resolution of a microcapillary array. In order to obtain a local light emission, non-radiative processes must dominate the energy transfer from solvent to dye [26, 39]. The probability $(\eta)$ of non-radiative energy transfer increases with dye concentration according to the expression [40] $\eta=C /\left(C+C_{0}\right)$. For the LS's considered $C_{0}$ ranges between 1.0 and $1.6 \mathrm{~g} / 1$, and dye concentrations larger than these values are necessary to ensure a high locality of light emission. However, too high a concentration value would result in significant light attenuation along the capillaries, despite the large Stokes shift (Fig. 5) that is a common feature of the LS's considered. The best compromise between the requirements of high scintillation efficiency, local light emission and low attenuation is reached at a concentration of $\sim 3 \mathrm{~g} / 1$ for all dye-solvent combinations tested.

\section{- High refractive index}

A high refractive index is needed to maximize the fraction of the scintillation light trapped in the capillary in which it is produced. This trapping fraction depends on the refractive index $n_{1}$ of the LS (i.e. the fibre core), on the refractive index $n_{2}$ of the capillary glass (i.e. the fibre cladding), and on the geometry of the fibre cross-section. For an ideal fibre having a circular cross-section, and assuming that light is produced isotropically and uniformly in the core, the trapping fraction in each direction along the fibre is [7] $\frac{1}{2}\left(1-n_{2}{ }^{2} / n_{1}{ }^{2}\right)$. The refractive indices of LS's based on $1 \mathrm{MN}$ and on IPN are respectively $n_{1}=1.62$ and $n_{1}=1.59$, significantly higher than the refractive index of the capillary glass, $n_{2}=1.49$. In the case of the ideal fibre of circular cross-section, the resulting trapping fraction in each direction of the fibre is $7.7 \%$ for LS's based on $1 \mathrm{MN}$ and $6.1 \%$ for LS's based on IPN.

\section{- Short decay time}

For all considered LS's based on $1 \mathrm{MN}$ or IPN, about $96 \%$ of the emitted light decays with a time constant of $\sim 6-8 \mathrm{~ns}$, and the remaining $4 \%$ of the light has a decay time of $\sim 30-40$ ns [30]. As an example, the decay curve of $1 \mathrm{MN}$ doped with R6 is shown in Fig. 7. The curve was obtained using the single-photoelectron delayed-coincidence counting method [41].

\section{- High radiation hardness}

In Fig. 8 we show the dependence of the scintillation yield on the absorbed dose for 1 MN doped with R6, R39 and R45 [37]. With the first two dyes the behaviour is quite similar and an absorbed dose as high as 200 Mrad reduces the light yield by only $\sim 30-40 \%$. For $1 \mathrm{MN}$ doped with R45, the sharp decrease of the light yield observed at a dose below $10 \mathrm{Mrad}$ is due to degradation of the dye [26,39]. The radiation hardness of LS's based on IPN can be even better [37] than that of LS's based on 1MN. For comparison, the behaviour of two LS's produced by Bicron $^{5}$ is also shown in Fig. 8. As well as affecting the LS, the dose absorbed by a capillary bundle can darken the capillary glass. Taking into account that, at each total internal reflection, light penetrates into the glass to a depth of a few wavelengths (evanescent wave), this glass darkening results in further light

\footnotetext{
${ }^{5}$ Bicron, Newbury, OH 44065, USA.
} 
attenuation, especially for rays that undergo many reflections before reaching the readout end of the bundle. This effect has been measured [37] and is found to be less important than the radiation damage of LS's, even for non-radiation-hard glass.

\section{- Bundle filling procedure}

The scintillation properties of our LS's depend strongly on their purity. In view of this, the LS's were purified before being used and steps were taken to avoid any contamination during the bundle filling procedure.

Various purification methods [42] have been set up. These have made possible drastic increases in the light yield and transparency of the LS's. As an example, we show in Fig. 9 the effect [26] of various degrees of purification on a LS based on IPN doped with R45. After three steps of purification the light yield is increased by a factor $\sim 1.8$ and the light attenuation length, measured in capillaries of $500 \mu \mathrm{m}$ diameter, is increased by a factor $\sim 9$.

The presence of reactive gases, in particular oxygen, dissolved in the LS's, is another cause of deterioration [38] of the scintillation properties. This is illustrated in Fig. 10, where we show the variation in the light yield of $1 \mathrm{MN}+\mathrm{R} 6$ when the scintillator is placed in atmospheres of different gases.

To avoid deterioration of the LS properties during the filling of a capillary bundle, we have developed the set-up represented in Fig. 11. With this set-up, the LS was first degassed in vacuum for several hours, and was then introduced into the bundle by applying a slight argon overpressure. A peristaltic pump forced the LS to circulate through the bundle for many hours, so as to eliminate gas bubbles that might be present in the capillaries, and which would cause poor light transmission. This procedure was continuously monitored by illuminating one end of the bundle and using a microscope to observe the light emerging from the other end. During the filling sequence the LS comes into contact with argon, and with glass, metals and teflon, all meticulously cleaned in advance.

Using the procedure described, the square bundle and the hexagonal bundle were filled with $1 \mathrm{MN}+3 \mathrm{M} 15$ and $1 \mathrm{MN}+\mathrm{R} 39$ respectively. In both cases the dye concentration was $3 \mathrm{~g} / 1$.

\section{Optoelectronic readout chain}

The light output for a minimum-ionizing particle crossing a capillary bundle perpendicularly to the bundle axis consists, at most, of a few photons per capillary, corresponding on average to less than one photoelectron. It is therefore necessary to amplify the output signal by a large factor before track images can be detected and stored using a positionsensitive receiver. The first stage of the readout chain is the most critical for the detection efficiency. It must have the highest possible quantum efficiency, including also the transmittance of the fibre-optic entrance window. Precisely engineered mechanical systems, such as shown in Fig. 12, ensure that there is good optical coupling between capillary bundle and first readout stage.

The image receiver adopted in the readout chain of the square bundle was a CCD made by Thomson ${ }^{6}$, model TH7896A-(H), composed of $1024 \times 1024$ pixels, each with

\footnotetext{
${ }^{6}$ Thomson, Division Tubes Electroniques, Boulogne-Billancourt, France.
} 
dimensions of $19 \times 19 \mu \mathrm{m}^{2}$. This CCD was preceded by a series of five Image Intensifiers $\left(\mathrm{II}_{1}-\mathrm{II}_{5}\right.$, Fig. 12), the first and the last made by Geosphaera, the others produced by Varo $^{7}$. These provided an amplification of $\approx 10^{5}$. The LS contained in the capillaries of the square bundle was in direct contact with the fibre-optic window of the first II. This II was chosen because of its high quantum efficiency $(\sim 20 \%$ at the wavelength of maximum sensitivity). Its demagnification ${ }^{8}(m=25 / 40 \simeq 0.62)$ was compensated by the second II, which magnified the image by a factor $m=46 / 25 \simeq 1.8$. All II's except the fourth were electrostatically focused. The main image intensification was provided by the fourth tube, which was proximity focused and contained a microchannel plate (MCP). The last II demagnified the image to fit the dimensions $\left(19.5 \times 19.5 \mathrm{~mm}^{2}\right)$ of the CCD. The spatial resolution of the II's of the chain, measured at a wavelength of 500 $\mathrm{nm}$, was $\sim 24 \mathrm{lp} / \mathrm{mm}$ for the MCP tube, and was in the range 29 to $39 \mathrm{lp} / \mathrm{mm}$ for the electrostatically focused II's.

The optoelectronic chain could be gated by applying a $\sim 200 \mathrm{~V}$ pulse to the photocathode of the MCP tube. This made a triggered readout possible. During the time required for the trigger decision $(\sim 180 \mathrm{~ns})$ the decay times of the phosphors of the first three II's provided an optical memory, temporarily storing track images. Most of the image persistence was introduced by the first II, which had a K-67 (green) phosphor, with a $\sim 10 \mu$ s decay time. The P47 (blue) phosphors of the second and third II's had a decay time of $\sim 35 \mathrm{~ns}$, and so did not contribute significantly to the optical memory. As shown in Fig. 13, the image persistence was sufficiently long that the wait for the first-level trigger resulted in the loss of only a small fraction of the light. The fourth and fifth II's had, respectively, a P46 (green) phosphor, with a decay time of $\sim 80 \mathrm{~ns}$, and a KO-425 (blue) phosphor, with a decay time of $\sim 50 \mathrm{~ns}$. Since these phosphors came after the MCP, where the trigger was applied, they did not contribute to the optical memory.

The readout chain of the hexagonal bundle (Fig. 12) had an EBCCD camera as image receiver, and was much more compact than the readout chain described above. The EBCCD [43-45] combined the functions of an electrostatically focused II and a conventional CCD. It was essentially an II where the phosphor screen was replaced by a reversed thinned CCD (thickness $\sim 10 \mu \mathrm{m}$ ), consisting of $1024 \times 1024$ pixels, each with dimensions of $13 \times 13 \mu \mathrm{m}^{2}$. Electrons emitted at the photocathode were accelerated through $\sim 15 \mathrm{kV}$ toward the CCD, where one electron-hole pair was created for every $3.6 \mathrm{eV}$ released in the silicon. A gain of $\sim 3500$ electrons/photoelectron was obtained [43]. Using specially designed low-noise readout electronics, a single photoelectron was clearly distinguished from the background. The EBCCD then provided all the signal amplification required to allow detection of track images. Nonetheless, the EBCCD was preceded in the readout chain by an II similar to the first II of the readout chain used with the square bundle. The II had the advantage of a high quantum efficiency, and the long decay time of the II phosphor provided the optical memory while waiting for a trigger decision. When the trigger was satisfied, the EBCCD was gated by applying a $1.6 \mathrm{kV}$ pulse to the focusing electrode. The optical coupling to the readout chain for the hexagonal bundle was slightly different from that for the square bundle. A fibre-optic plate (Fig. 12) was fixed to the bundle's end, then the entire structure was coupled to the entrance window of the II. This meant that the LS was more securely sealed, but the detected light was reduced by $\sim 40 \%$ because of losses in the fibre-optic plate.

\footnotetext{
${ }^{7}$ Varo Inc., Garland, TX 75041, USA.

${ }^{8}$ The magnification $m$ of a non-zoomable image intensifier is reported as the ratio of the output diameter to the input diameter, all diameters in $\mathrm{mm}$.
} 


\section{Set-up for tests}

The performance of the two large capillary bundles has been studied in a pilot experiment located in front of the CHORUS detector [27], in the CERN wideband neutrino beam. In this situation the bundles could detect either beam muons or neutrino interactions.

For muon tracking, the bundles were placed perpendicularly to the beam direction. Muons were selected by a $2.5 \times 5 \mathrm{~cm}^{2}$ trigger counter placed in front of the bundles.

For studies of neutrino interactions (Fig. 14), the square bundle was placed vertically, so that it was perpendicular to the beam (transverse configuration), while the hexagonal bundle was placed horizontally, with its axis parallel to the beam (longitudinal configuration). In the images recorded with the transverse configuration, most of the outgoing tracks from neutrino interactions in the bundle were collimated in the forward direction, within approximately $\pm 150 \mathrm{mrad}$. In the longitudinal configuration, by contrast, tracks of interaction products were spread over $2 \pi \mathrm{rad}$, in an azimuthal projection.

Neutrino interactions in the capillary bundles were detected by two plastic scintillator counters positioned immediately downstream of the bundle (Fig.14). Counters $\mathrm{S}_{1}$ and $\mathrm{S}_{2}$, used with the square bundle, had dimensions of $160 \times 2.5 \mathrm{~cm}^{2}$; counters $\mathrm{S}_{3}$ and $\mathrm{S}_{4}$, used with the hexagonal bundle, had dimensions of $20 \times 20 \mathrm{~cm}^{2}$. Tracks that were measured in the capillary bundles and crossed the CHORUS emulsion target could be reconstructed in the various downstream detectors.

The trigger logic for detecting neutrino interactions is represented in Fig. 15. A firstlevel "local" trigger was satisfied when there was a coincidence between $\mathrm{S}_{1}$ and $\mathrm{S}_{2}$ (or between $\mathrm{S}_{3}$ and $\mathrm{S}_{4}$ for the hexagonal bundle) during the $6 \mathrm{~ms}$ accelerator spill. The firstlevel local trigger occurred $\sim 180 \mathrm{~ns}$ after the physical event. Subsequently, a $1 \mu$ s pulse opened the gate of the MCP (or of the EBCCD), allowing intensified images to pass to the CCD (or to the silicon chip in the EBCCD). The gate pulse defined the time to wait for a second-level trigger from the CHORUS trigger system. This included a large veto counter, placed in front of the apparatus (Fig. 14), for rejection of beam muons. If this second-level trigger arrived within $1 \mu$ s of the first-level trigger, the gate of the MCP (or EBCCD) was kept open for an additional $100 \mu \mathrm{s}$, a time long enough to enable collection at the CCD chips of most of the scintillation light produced in the capillary bundle and intensified by the optoelectronic chain.

Since neither the CCD nor the EBCCD had a fast-clear facility, these sensors were cleared by continuous readout at a pixel clock frequency of $10 \mathrm{MHz}$. This means that the chips were fully cleared every $\sim 110 \mathrm{~ms}$. When a first-level trigger arrived, the continuous clearing was interrupted and the image integration at the silicon chip was enabled by an integration-time control (ITC) pulse of $\sim 200 \mu \mathrm{s}$. If the first-level trigger was confirmed by the second-level trigger, an interrupt (INTR) signal began the data acquisition, during which a dead time (DT) prevented further triggers. The timing of all pulses relevant to readout is indicated in Fig. 15.

\section{$7 \quad$ Experimental results}

\subsection{Magnification and distortion of the readout chains}

The magnification of an electrostatically focused II usually has a slight dependence on the distance between the point considered and the centre of the photocathode. The result is pincushion distortion: the image of a radial straight line is still straight and radial, but the image af an off-centre line appears curved. This effect was appreciable for the square 
bundle's readout chain, which contained 4 electrostatically focused II's ${ }^{9}$, but was found to be negligible for the readout chain of the hexagonal bundle.

To have quantitative measures of the distortion with each bundle and readout chain, a reference grid was placed in contact with the bundle end opposite the readout window. This grid, which consisted of $20 \mu \mathrm{m}$ width transparent lines at $2 \mathrm{~mm}$ intervals, was illuminated from behind by an LED. In Fig. 16 we show the image of the grid as seen by the CCD through the readout chain used with the square bundle. The distortion affecting the image must be taken into account if the directions of particles crossing the bundle are to be correctly reconstructed. The distortion was parameterized using the expression $R_{o}=\left(R_{i} / m\right)\left(1+a R_{i}+b R_{i}{ }^{2}\right)$, where $R_{o}$ and $R_{i}$ are the respective distances from the optical centre of a point in the object (grid) space and of a point in the image (CCD) space, $m$ is the "central" magnification of the chain (i.e. the magnification of the chain along its axis), and $a$ and $b$ are coefficients. First, the coordinates of the optical centre were found by taking advantage of the radial symmetry of the distorted grid image. Next, the values of $m, a$ and $b$ were determined from the knowledge of the distance between grid lines in the object space. In the case of the square bundle's readout chain we found that the optical axis was displaced with respect to the bundle centre by $\sim 2 \mathrm{~mm}$, that the central magnification was $m \simeq 1.24$, close to the nominal value of 1.27 , and that the two parameters describing the distortion had values $a \simeq-2 \times 10^{-3} \mathrm{~mm}^{-1}$ and $b \simeq-3 \times 10^{-4} \mathrm{~mm}^{-2}$. Once these parameters had been determined, all distortion effects could be corrected for. The distortion correction improved the efficiency for track finding in off-line analyses, and allowed better definition of track directions. The width of the distribution of hit displacements from fitted tracks (residuals distribution), which is typically a few tens of $\mu \mathrm{m}$, is reduced by $\sim 22 \%$ when the correction is applied.

\section{$7.2 \quad$ Vignetting}

Like the magnification, the light amplification of an electrostatically II is usually dependent on the distance between the point considered and the optical axis of the tube. This is essentially a consequence of the curvature of the internal surfaces of the fibre-optic input and output windows. This curvature implies larger glass thickness, and so lower light transmission, at the window edges. This "vignetting" effect can introduce gain nonuniformity of $20-50 \%$ for electrostatically focused II's. In proximity focused II's, where the input and output windows are flat, this effect is not present and the gain is uniform to within a few percent over the full photocathode surface.

The effect of vignetting was expected to be important for the square bundle's readout chain, where 4 electrostatically focused II's were present, and has been studied [46] by analyzing beam muons crossing the bundle perpendicularly to its axis. The transverse dimension of the beam was large enough to ensure that the triggering muons were uniformly distributed across the bundle. However, not all of them were recorded because only the central part of the bundle was seen by the II chain and by the CCD (Fig. 17).

More than a thousand muon tracks were recorded. In the offline analysis of these events a threshold was first applied to the CCD pixel pulse height, so as to reduce the background, then muon tracks were reconstructed. In Fig. 17a) we show, in the projection perpendicular to the bundle axis, the positions of all hits lying on the reconstructed tracks. The applied threshold resulted in a loss of hits near the edges of the readout zone, where the light gain of the chain was lowered by the vignetting. The effect is even more evident in Fig. 17b), where we show the pulse height distribution measured by the CCD.

\footnotetext{
${ }^{9}$ The MCP tube was proximity focused, and so did not contribute significantly to the distortion.
} 


\subsection{Hit density and light attenuation}

To evaluate the tracking capabilities of the two capillary bundles, images produced by minimum-ionizing particles crossing perpendicularly to the bundles were analyzed. For each trigger, coordinates and pulse heights were recorded for all pixels with pulse height above a threshold value. A tuning of the pulse height threshold was performed as the first step of the offline analysis. We then formed clusters from contiguous pixels of abovethreshold pulse height, centred on a local maximum (a pixel with a larger pulse height than the 8 surrounding pixels). A track appeared as a succession of approximately aligned clusters. Each cluster comprised several tens of pixels and corresponded to a photoelectron emitted at the photocathode of the first II of the readout chain. A cluster's centroid was calculated as the weighted average of the pixel coordinates, the weights being the pixel pulse heights. Each cluster centroid was identified as a hit. Taking into account magnification and distortion in the readout chain, so that distances were referred back to the real space of the detector, hit positions were reconstructed in a plane orthogonal to the bundle axis. A straight line was fitted to the tracks, using only hits within a road of $\pm 150 \mu \mathrm{m}$.

Light attenuation along the capillaries resulted in the hit density along a track depending on the distance, $d$, between the track and the readout end of the bundle. For the square bundle, measurements of hit density were performed just after filling, in tests in a $5 \mathrm{GeV}$ pion beam. Measurements were repeated, for muon tracks, at intervals over a two-year period. During this time the LS in the bundle was left unchanged and the entire detector, with its readout chain, was mounted in the cool box $\left(\sim 5{ }^{\circ} \mathrm{C}\right)$ of the CHORUS experiment [27]. The results of these measurements ${ }^{10}$ are shown in Fig. 18. No ageing effect is present over the two years. All points are well fitted by the sum of only two exponentials. The light's attenuation length (Fig. 18) varies from $\lambda \simeq 50 \mathrm{~cm}$ near the bundle's readout end, to $\lambda \simeq 400 \mathrm{~cm}$ at the opposite end. Extrapolating the fit to $d=0$ leads to a maximum hit density of $\approx 8$ hits $/ \mathrm{mm}$.

For the hexagonal bundle, measurements have been made for only four distances, and do not allow a meaningful determination of the attenuation length. The lower hit density measured for this bundle is mostly due to the fibre-optic plate added between the bundle's readout end and the optoelectronic chain, as described in Section 5. Other factors, such as the quantum efficiencies of the first two photocathodes of each readout chain, and the quality of the inner surfaces of the capillaries could also contribute to the different hit densities.

\subsection{Spatial resolution from single-track events}

We have measured the spatial resolution of our detectors through an analysis of the tracks of minimum-ionizing particles. As well as being influenced by physical effects, such as delta-ray production and Coulomb scattering, the spatial resolution of a detector based on glass capillaries is dependent on several factors. These include the inner diameter of the capillaries, the uniformity of the capillary array, and the resolution and distortion of the optoelectronic chain.

The spatial resolution can be described in terms of three quantities. These are the "cluster size" $\left(\sigma_{c s}\right)$, the "track residual" $\left(\sigma_{t r}\right)$ and the "two-track resolution" $\left(\sigma_{t t}\right)$. The square of the last-mentioned quantity is approximately the sum of the squares of the other

\footnotetext{
${ }^{10}$ The present results are slightly different from those reported in references [25] and [47]. An error in the data analyses reported in these previous works has now been corrected.
} 
two. All measurements made are with reference to a two-dimensional image projection on a plane perpendicular to the bundle. Unless indicated otherwise, values are referred to the real space of the bundle: image magnification, including the magnification in the tapered section of the square bundle, is taken into account.

\section{- Cluster size}

An isolated cluster was due to a single photoelectron emitted at the photocathode of the first II of the optoelectronic chain. Its size on the CCD was therefore unaffected by the bundle characteristics and depended only on the spatial resolution of the components of the readout chain. The average cluster profile has been obtained by superposing the centroid of many isolated clusters and summing the pulse heights of the pixels at each position. This profile was then projected onto an axis, chosen parallel to the pixel sides. Resulting distributions for the two readout chains are shown in Fig. 19a. These distributions are fitted by a Gaussian superimposed onto a broad background. The standard deviations of the Gaussians provide measures of the cluster size. The values obtained are $\sigma_{c s} \simeq 21 \mu \mathrm{m}$ for the square bundle and $\sigma_{c s} \simeq 26 \mu \mathrm{m}$ for the hexagonal bundle, in reasonable agreement with the values of $20 \mu \mathrm{m}$ and $22 \mu \mathrm{m}$ expected from the nominal parameters of the readout chains. If, instead of being referred to the real space of the bundle, the cluster sizes are evaluated at the $\mathrm{CCD}$, the values obtained are $\sigma_{c s}^{\mathrm{CCD}} \simeq 27 \mu \mathrm{m}$ with the square bundle and $\sigma_{c s}^{\mathrm{CCD}} \simeq 14 \mu \mathrm{m}$ with the hexagonal bundle, reflecting the better spatial resolution of the readout chain composed of only one II followed by the EBCCD tube.

\section{- Track residuals}

Track residuals measure hit displacements from fitted tracks. Since each hit is assumed to correspond to a single photoelectron, all hit displacements are given the same weight. In Fig. 19b we show distributions of track residuals for particles crossing the capillary bundles at a fixed distance from the readout end. These distributions have been fitted by a Gaussian superimposed onto a broad background. The values obtained for the standard deviations of the Gaussians are $\sigma_{t r} \simeq 27 \mu \mathrm{m}$ for the square bundle and $\sigma_{t r} \simeq 33 \mu \mathrm{m}$ for the hexagonal bundle. These values are significantly larger than expected for an ideal capillary bundle. A simulation that takes into account the capillary diameter, the resolution of the image intensifiers and the finite CCD pixel size predicts a value of $\sigma_{t r}$ in the range $12-15 \mu \mathrm{m}$ for the two bundles. This suggests that a significant contribution to the spatial resolution comes from the non-uniformity of the large-volume capillary bundles. This view is supported by the finding [47] that $\sigma_{t r}$ varies by about $\pm 10 \%$ for tracks crossing the square bundle at different positions along its length.

\section{- Two-track resolution}

The combined effect of the cluster size and of hit displacements is seen in the distribution of pulse height about fitted tracks. Distributions measured for particles at a fixed distance from the bundles' readout end are shown in Fig. 19c. The distributions have again been fitted by a Gaussian superimposed onto a broad background. The standard deviations of the Gaussians represent the detectors' two-track resolution. The values obtained are $\sigma_{t t} \simeq 34 \mu \mathrm{m}$ for the square bundle and $\sigma_{t t} \simeq 42 \mu \mathrm{m}$ for the hexagonal bundle. Like $\sigma_{t r}, \sigma_{t t}$ has been found [47] to vary along the length of the square bundle, the variation in this case being about $\pm 5 \%$. 
A comparison of the two distributions of Fig. 19c shows that the detector based on the square bundle had a better two-track resolution than the detector based on the hexagonal bundle. The magnification introduced by the tapered end of the square bundle compensates for the worse resolution of the bundle's readout chain.

\subsection{Reconstruction of many-track events}

The good tracking capability of the capillary technique is illustrated in Fig. 20, where we show four examples of neutrino interactions, two in the square bundle placed perpendicularly to the beam, and two in the hexagonal bundle placed longitudinally to the beam.

For events recorded with the transverse configuration (square bundle), various methods allowing determination of the resolution on vertex position and on track direction have been developed [48]. After an approximate determination of the vertex position, all hit combinations are tested to form track candidates roughly consistent with an origin at the vertex. A circular projection algorithm is used to perform the final track selection. Each track is then represented by a straight line fitted to hits within a road of $\pm 150 \mu \mathrm{m}$. Hits lying in the confused region very close to the vertex are not considered. Tracks are fitted to a single vertex, and the error matrix for the vertex parameters allows definition of the resolution on vertex position. Using this automatic fitting procedure, several tens of neutrino interactions have been analyzed. The resolution on vertex position was found to be $\sim 30 \mu \mathrm{m}$ in the direction orthogonal to the beam and $\sim 90 \mu \mathrm{m}$ along the beam. The perpendicular distance between a track and the vertex defines the track's impact parameter. A track with an impact parameter significantly different from zero may indicate the presence of a short-lived particle among the interaction products. The distribution of impact parameters is nearly exponential, with a mean value of $\sim 43 \mu \mathrm{m}$.

Tools have also been developed to allow the reconstruction described above to be performed under the guidance of an operator interacting with a graphics display. With the interactive fit, the reconstruction efficiency is increased by $\sim 14 \%$. By selecting well-defined, distortion-free track segments, a mean impact parameter of $\sim 23 \mu \mathrm{m}$ has been obtained.

The potential of the capillary target for detection of short-lived particles is illustrated by the event shown in Fig. 21. This event features five charged particles emerging from a neutrino interaction in the capillary bundle. Three of the particles were detected in the CHORUS apparatus, while the other two were emitted outside its angular acceptance. Two of the three particles detected in CHORUS were identified in the muon spectrometer as opposite-sign muons, while the third particle was detected in the fibre tracker. Whereas the $\mu^{-}$comes from the neutrino interaction vertex, close inspection of the $\mu^{+}$track shows that it comes from the decay of a short-lived particle. The projected decay kink is very small, but the decay can be identified from a change in the apparent hit density at the decay point, this change resulting from the difference in angle between the decaying particle and the $\mu^{+}$. The short black track emerging from the primary vertex could be either a nuclear fragment or a track emitted almost parallel to the capillaries. The energy of the $\mu^{+}(50 \mathrm{GeV})$ and the projected flight path of the decaying particle are consistent with the decay $D^{+} \rightarrow \bar{K}^{0} \mu^{+} \nu_{\mu}$, the $\bar{K}^{0}$ showing up as a $\sim 70 \mathrm{GeV}$ shower in the hadronic calorimeter. 


\section{Conclusions}

We have developed high-resolution tracking detectors based on glass capillaries filled with organic liquid scintillators of high refractive index. Capillary detectors are an evolution of detectors based on glass or plastic scintillating microfibres and offer an improved

performance. Such tracking devices can be of great interest in high-energy physics as microvertex detectors for the study of vertex topologies and decays of short-lived particles.

Two large-size prototype detectors have been constructed. The two capillary bundles, each composed of $\approx 10^{6}$ capillaries, were manufactured with two different procedures, and were operated with different readout chains. One of the readout chains was based on Image Intensifiers read by a standard CCD, while the other took advantage of the newly developed EBCCD tube. In both systems, images were detected by $\sim 10^{6}$ pixels.

A high scintillation efficiency and a light-attenuation length in excess of $3 \mathrm{~m}$ has been achieved through special purification of the liquid scintillators used. Along the tracks of minimum-ionizing particles, the hit densities obtained were $\sim 8 \mathrm{hits} / \mathrm{mm}$ at the readout window, and $\sim 3 \mathrm{hits} / \mathrm{mm}$ at a distance of $\sim 1 \mathrm{~m}$.

The two capillary-based detectors have been operated in front of the CHORUS detector, in the CERN wide-band neutrino beam. Observation of muon tracks and neutrino interactions has allowed evaluation of the track and vertex reconstruction capabilities. Typical spatial resolutions are in the range $20-40 \mu \mathrm{m}$.

\section{Acknowledgements}

This work has been carried out in the framework of the CERN Research and Development programme RD46. Finacial support was provided through the European Union's TMR Network Contract ERBFMRX-CT98-0196.

\section{References}

[1] R. Ruchti et al., IEEE Trans. Nucl. Sci. NS-32 (1985) 590.

[2] R. Ruchti et al., IEEE Trans. Nucl. Sci. NS-33 (1986) 151.

[3] M. Atkinson et al., Nucl. Instr. and Meth. A254 (1987) 500.

[4] M. Atkinson et al., Nucl. Instr. and Meth. A263 (1988) 333.

[5] C. Angelini et al., Nucl. Instr. and Meth. A277 (1989) 132.

[6] C. Angelini et al., Nucl. Instr. and Meth. A289 (1990) 342.

[7] C. Angelini et al., Nucl. Instr. and Meth. A295 (1990) 299.

[8] H. Blumenfeld et al., Nucl. Instr. and Meth. A278 (1989) 619.

[9] C. D'Ambrosio et al., Nucl. Instr. and Meth. A325 (1993) 161.

[10] M. Adinolfi et al., Proc. of the Workshop on Application of Scintillating Fibers in Particles Physics, Blossin, Germany, September 1990, ed. by R. Nahnhauer.

[11] M. Adinolfi et al., Nucl. Instr. and Meth. A310 (1991) 485. 
[12] M. Adinolfi et al., Nucl. Instr. and Meth. A315 (1992) 67.

[13] H. Leutz, Nucl. Instr. and Meth. A364 (1995) 422, and references therein.

[14] A.G. Denisov et al., Nucl. Instr. and Meth. A310 (1991) 479.

[15] A. Artamonov et al., Nucl. Instr. and Meth. A300 (1991) 53.

[16] N.I. Bozhko et al., Nucl. Instr. and Meth. A317 (1992) 97.

[17] M. Adinolfi et al., Nucl. Instr. and Meth. A311 (1992) 91.

[18] M. Adinolfi et al., Nucl. Instr. and Meth. A315 (1992) 177.

[19] C. Cianfarani et al., Nucl. Instr. and Meth. A339 (1994) 449.

[20] A. Cardini et al., Nucl. Instr. and Meth. A346 (1994) 163.

[21] S. Buontempo et al., Nucl. Instr. and Meth. A360 (1995) 7.

[22] P. Annis et al., Nucl. Instr. and Meth. A367 (1995) 377.

[23] G. Martellotti et al., Proc. Int. Soc. Opt. Eng., SPIE 2551 (1995) 53.

[24] P. Annis et al., Nucl. Phys. B (Proc. Suppl.) 54B (1997) 86.

[25] P. Annis et al., Nucl. Phys. B (Proc. Suppl.) 61B (1998) 390.

[26] V.G. Vasil'chenko et al., Instr. and Exp. Tech. 40 (1997) 175.

[27] E. Eskut et al. (CHORUS collaboration), Nucl. Instr. and Meth. A401 (1997) 7.

[28] R. van Dantzig et al., RD46 Proposal, CERN/LHCC 95-7, P60/LDRB, February 1995.

[29] C. Angelini et al., Nucl. Instr. and Meth. A281 (1989) 50.

[30] G.I. Britvich et al., Nucl. Instr. and Meth. A425 (1999) 498.

[31] C. Angelini et al., Nucl. Instr. and Meth. A289 (1990) 356.

[32] M. Atkinson et al., Nucl. Instr. and Meth. A254 (1987) 500.

[33] P. Pavan et al., Nucl. Instr. and Meth. B61 (1991) 487.

[34] S. Majewski et al., Nucl. Instr. and Meth. A281 (1989) 497.

[35] G.I. Britvich et al., Nucl. Instr. and Meth. A326 (1993) 483.

[36] S. Majewski et al., Nucl. Instr. and Meth. A281 (1989) 500.

[37] S.V. Golovkin et al., Nucl. Instr. and Meth. A362 (1995) 283.

[38] S. Buontempo et al., Nucl. Instr. and Meth. A425 (1999) 494.

[39] S.V. Golovkin et al., Nucl. Instr. and Meth. A305 (1991) 385.

[40] J. B. Birks, The Theory and Practice of Scintillation Counting, Pergamon Press, Oxford 1964. 
[41] L.M.Bolinger and G.E.Thomos, Rev. Sci. Instr. 32 (1961) 1044.

[42] V.G. Lapshin et al., IHEP report n. 93-25, Protvino, 1993.

[43] S. Buontempo et al., Nucl. Instr. and Meth. A413 (1998) 255.

[44] P. Annis et al., Proc. of the Workshop on Scintillating Fiber Detectors, Notre Dame, USA, ed. by A.D. Bross, R.C. Ruchti and M.R. Wayne, p. 509.

[45] S.V. Golovkin et al., Proc. Int. Soc. Opt. Eng., SPIE 2551 (1995) 118.

[46] K. Holtz, Thesis, Westfälische Wilhelms-Universität, Münster, 1998.

[47] K. Hoepfner, Thesis, Humboldt-Universität, Berlin, 1996.

[48] K. Hoepfner et al., Nucl. Instr. and Meth. A406 (1998) 195. 


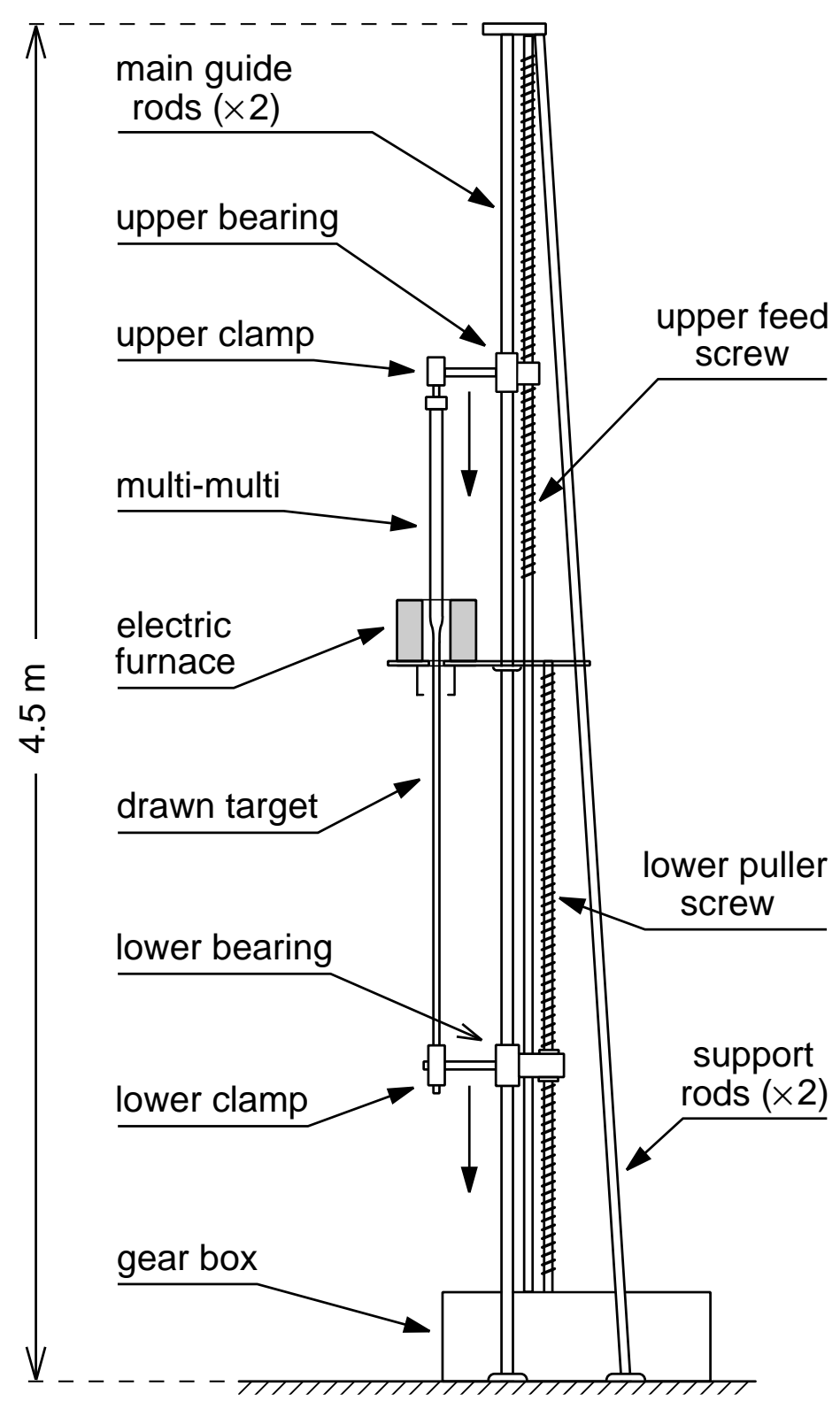

Figure 1: Schematic view of the tower used in the final drawing stage of the first manufacturing method described in the text. 


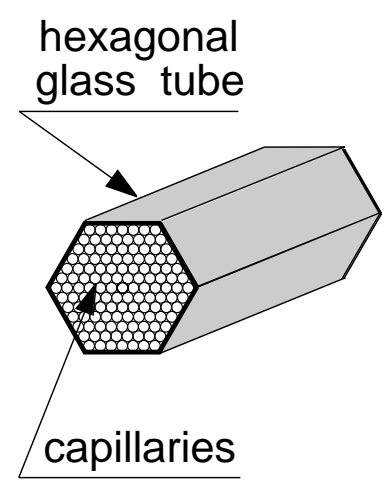

a)

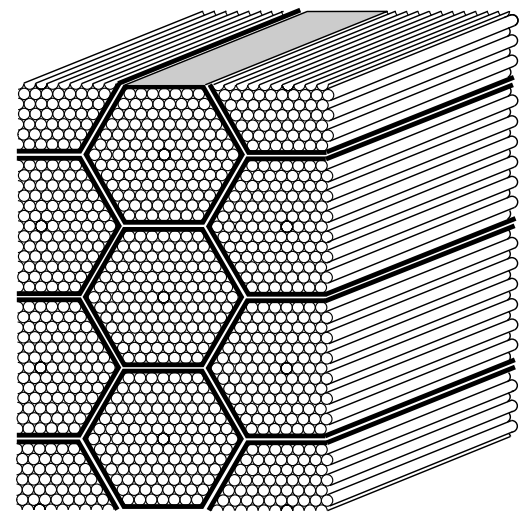

b)

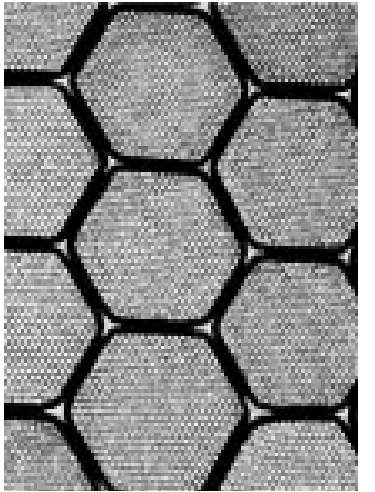

c)

Figure 2: Schematic view of a): a multi and b): the way in which multies are assembled in the final bundle. A microphotograph of a section of a typical capillary bundle is shown in c). The distance between opposite flat sides of a multi is $\sim 0.7$ (1.4) $\mathrm{mm}$ for the bundle manufactured with the first (second) process described in the text.
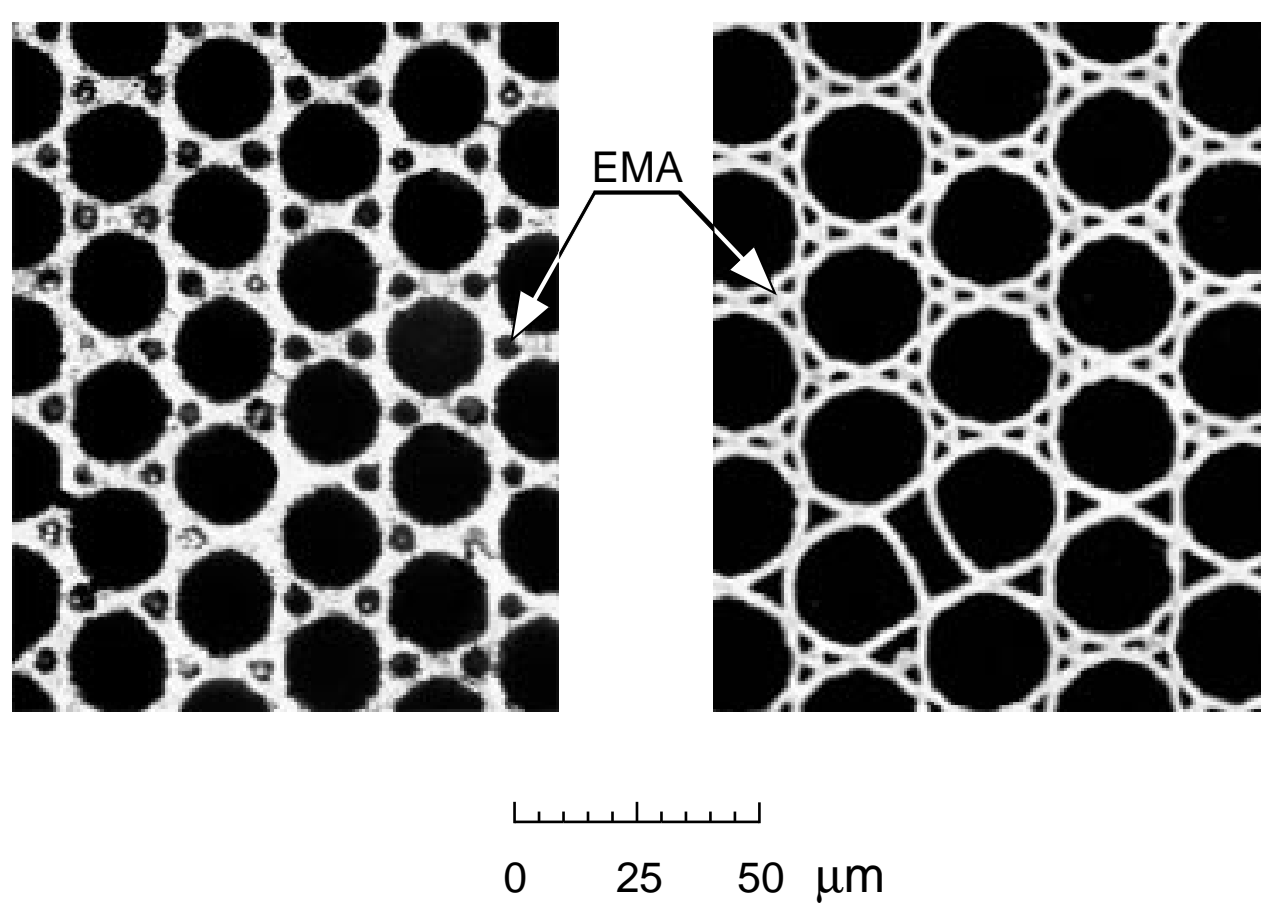

Figure 3: Microphotograph of capillary bundles manufactured with the first process (left) and with the second process (right), as described in the text. EMA is inserted in the interstices between capillaries. 


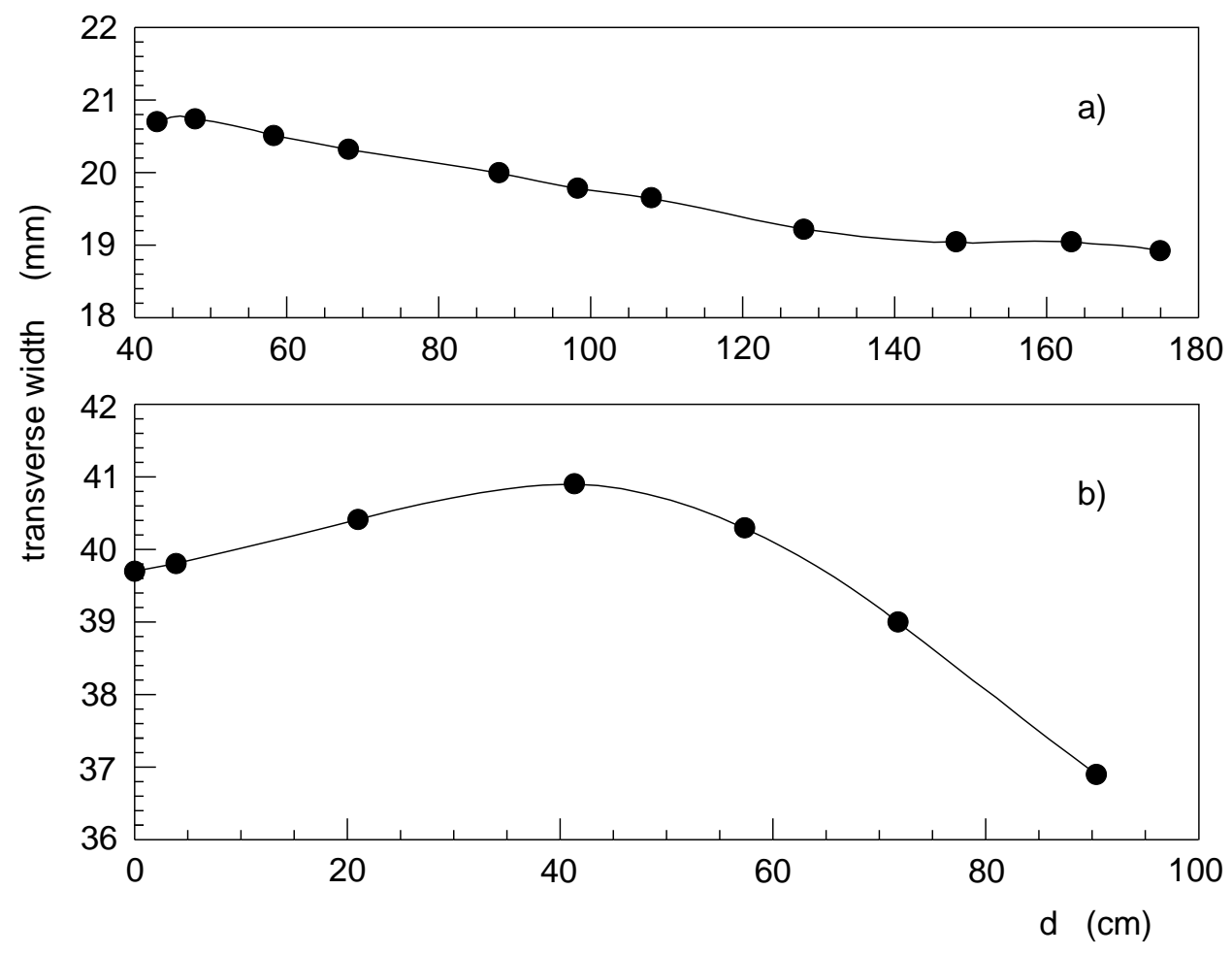

Figure 4: Transverse width (distance between opposite flat sides) measured for a) square bundle and b) hexagonal bundle, as a function of the distance $d$ from the readout end. The curves are drawn to guide the eye.

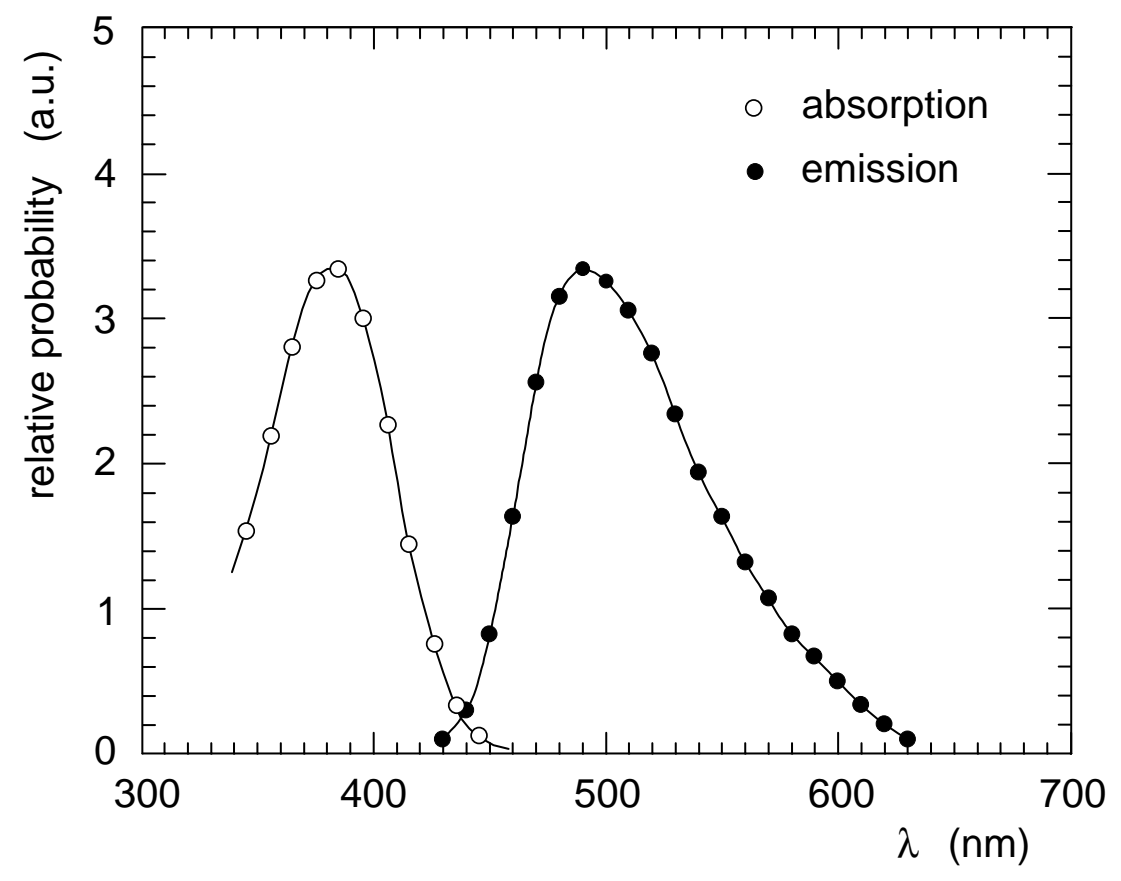

Figure 5: Absorption and emission spectra of $1 \mathrm{MN}+\mathrm{R} 6$. 


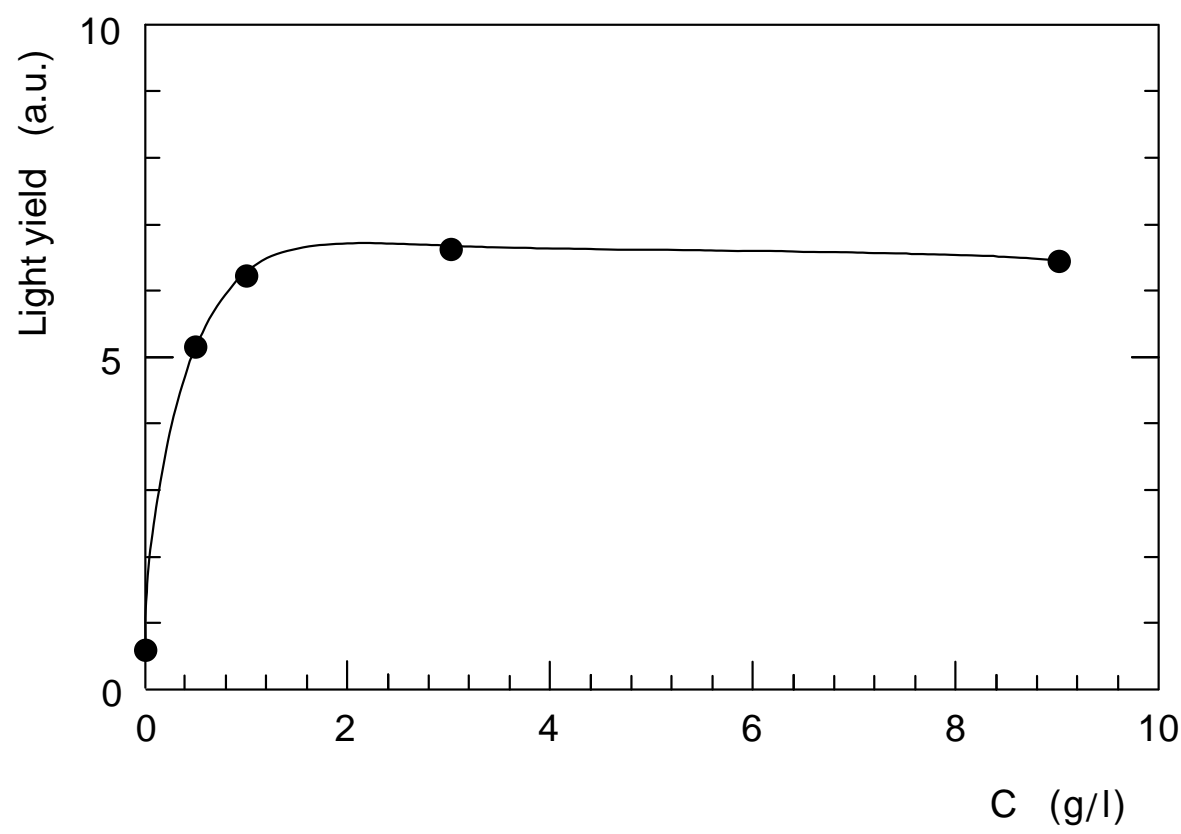

Figure 6: Light yield as a function of dye concentration, for a scintillator based on 1MN doped with R6. A similar behaviour has been observed for all of the scintillators based on $1 \mathrm{MN}$ or IPN.

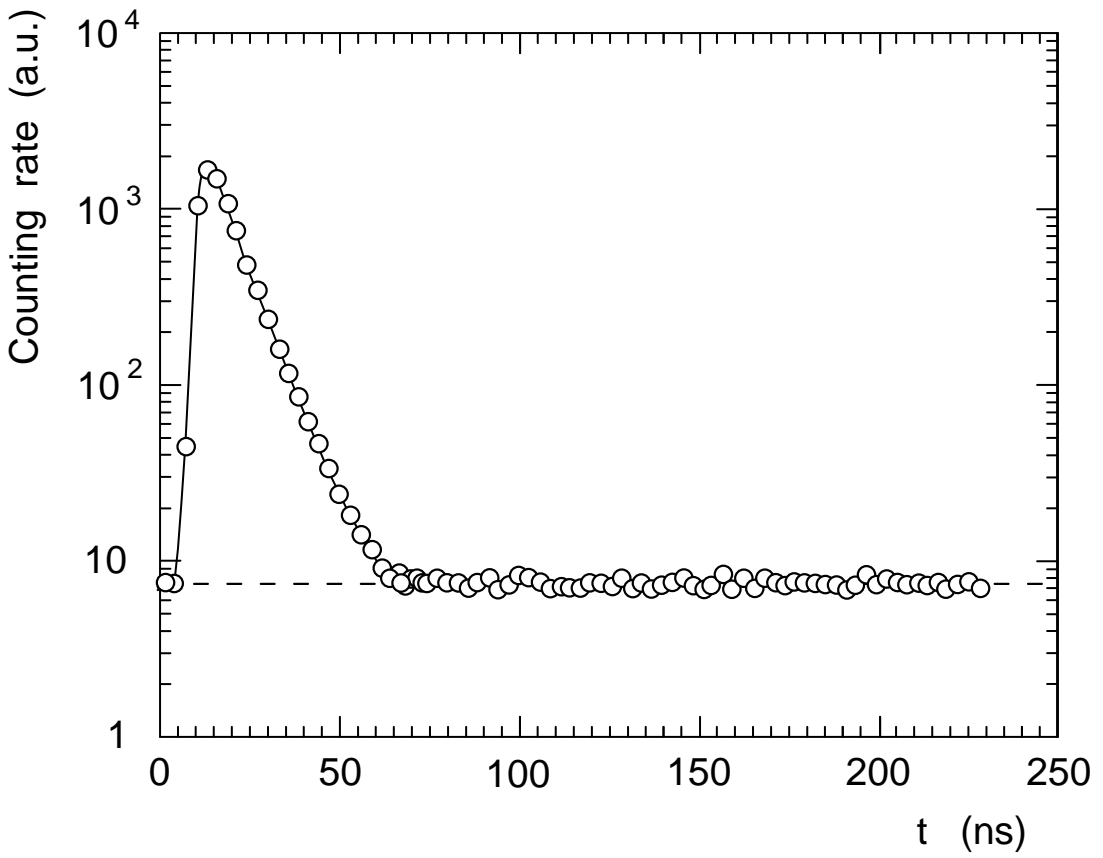

Figure 7: Emission time of liquid scintillator based on 1MN doped with R6. The measurements have been performed using the single-photoelectron delayed-coincidence counting method. The dashed line indicates the average rate of accidentals. Similar results have been obtained for all scintillators based on $1 \mathrm{MN}$ or IPN, and doped with R6, R39, R45 or $3 \mathrm{M}-15$. 


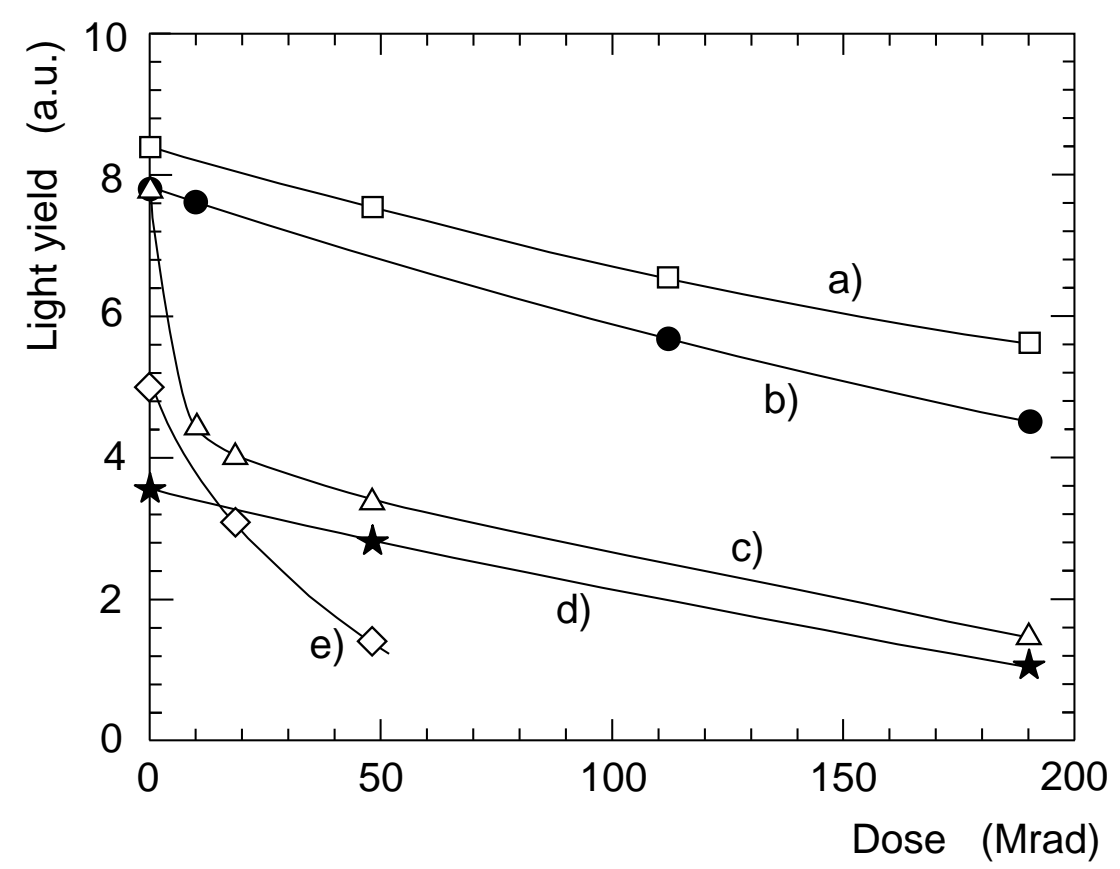

Figure 8: Light yield as a function of the absorbed dose, for 1MN doped with: a) R39; b) R6; c) R45; and for two LS's produced by Bicron: d) BC-599-13B; e) BC-599-13G.

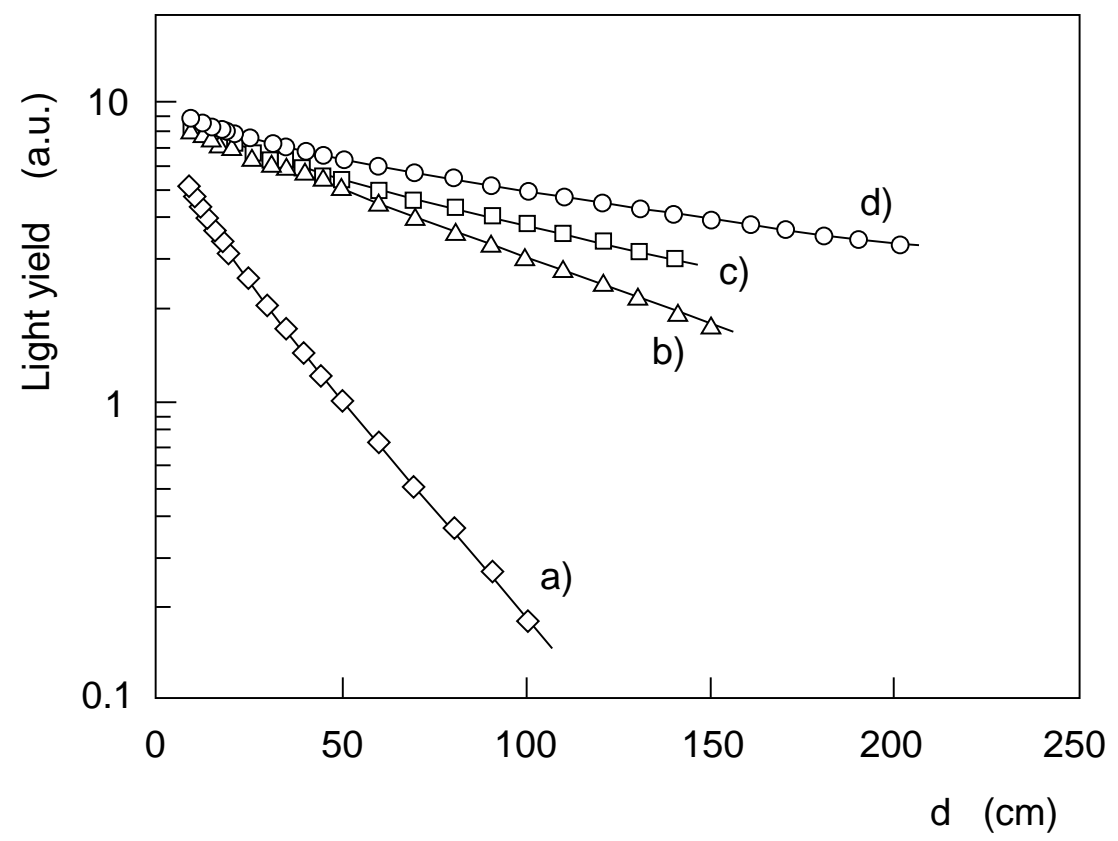

Figure 9: Light attenuation along capillaries that have $500 \mu \mathrm{m}$ diameter, and are filled with IPN+R45, for various degrees of scintillator purity: a) before purification; b) after one purification stage; c) after two stages; d) after three stages. 


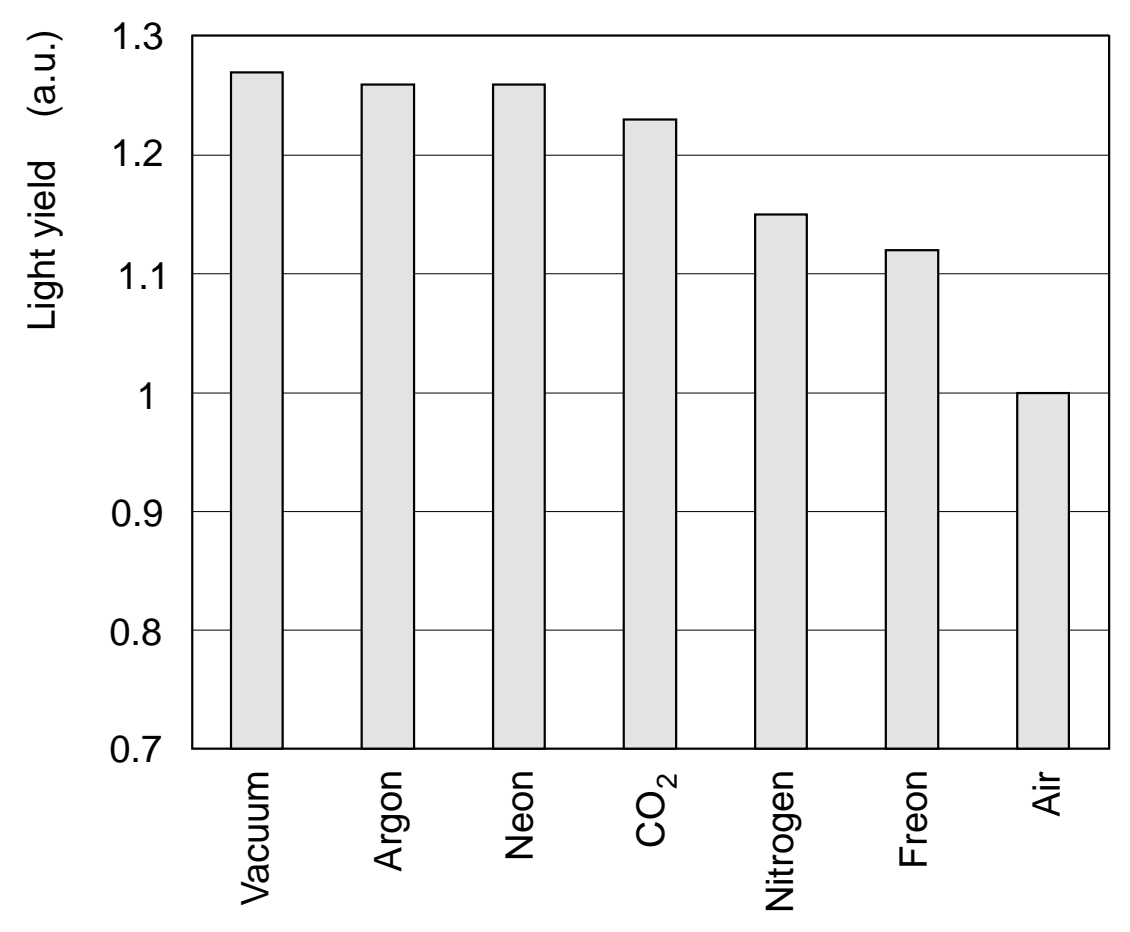

Figure 10: Light yield of $1 \mathrm{MN}+\mathrm{R} 6$ scintillator, when placed in atmospheres of different gases. The light yield in air is set to 1 .

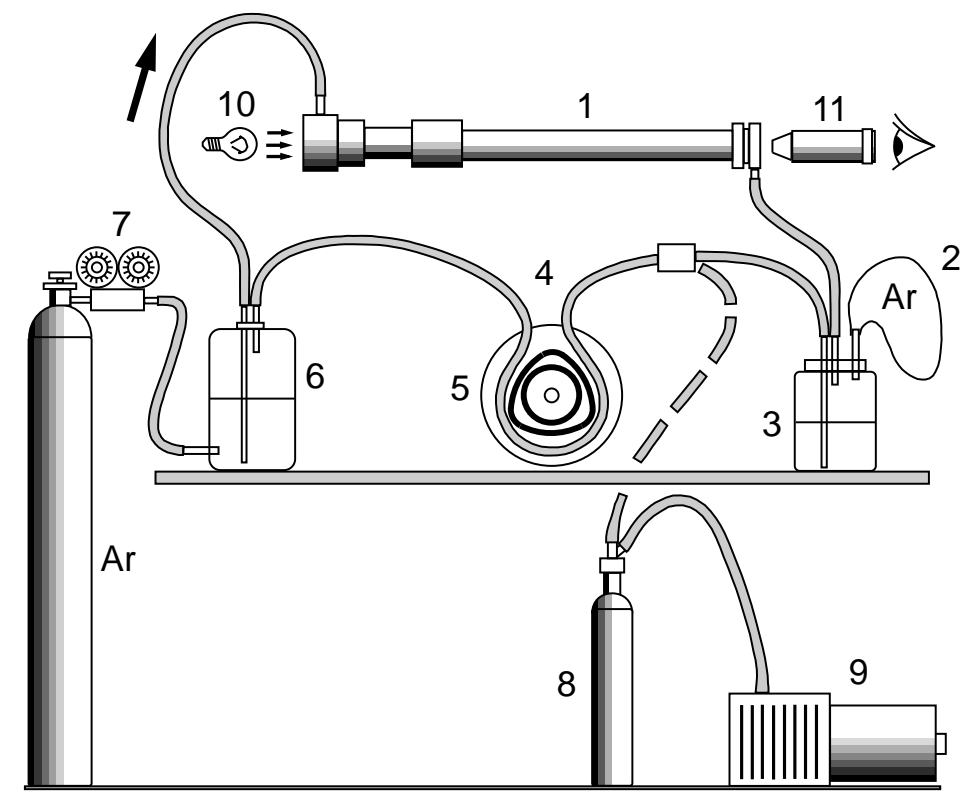

Figure 11: Schematic view of the set-up used for filling capillary bundles with LS: 1) capillary bundle; 2) Polyethylene balloon; 3) glass reservoir for LS; 4) soft hoses; 5) peristaltic pump; 6) glass vessel for introduction of Ar; 7) pressure-gauge; 8) glass vessel for initial LS degassing; 9) vacuum pump; 10) light source and 11) microscope for monitoring the bundle filling. 

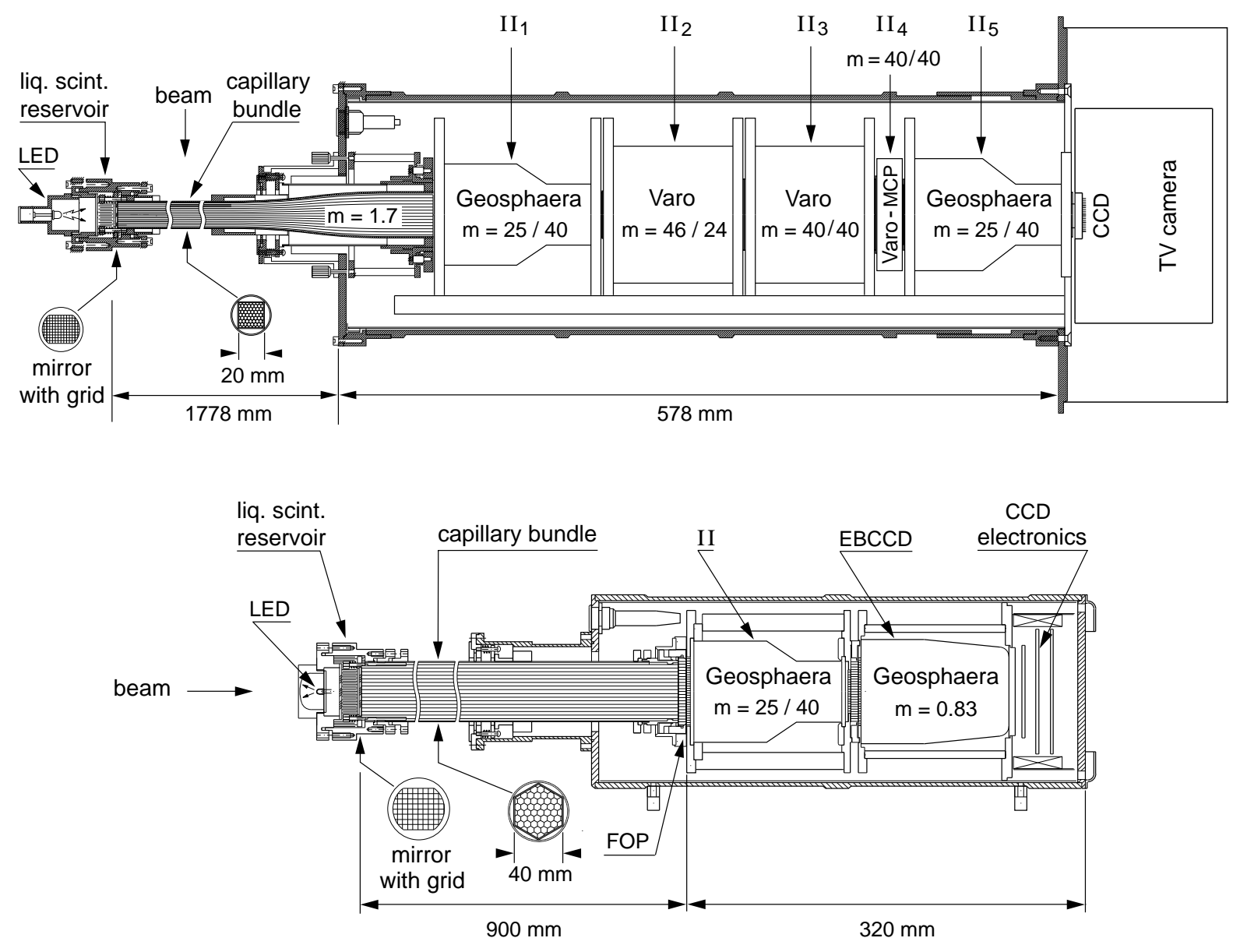

Figure 12: Optoelectronic readout chains coupled to the square capillary bundle (upper figure) and to the hexagonal capillary bundle (lower figure). The magnification $m$ of each non-zoomable II is expressed as the ratio of the output diameter to the input diameter (in $\mathrm{mm}$ ). The coupling to the readout chain is direct for the square bundle and is via a fibre-optic plate (FOP) for the hexagonal bundle. 


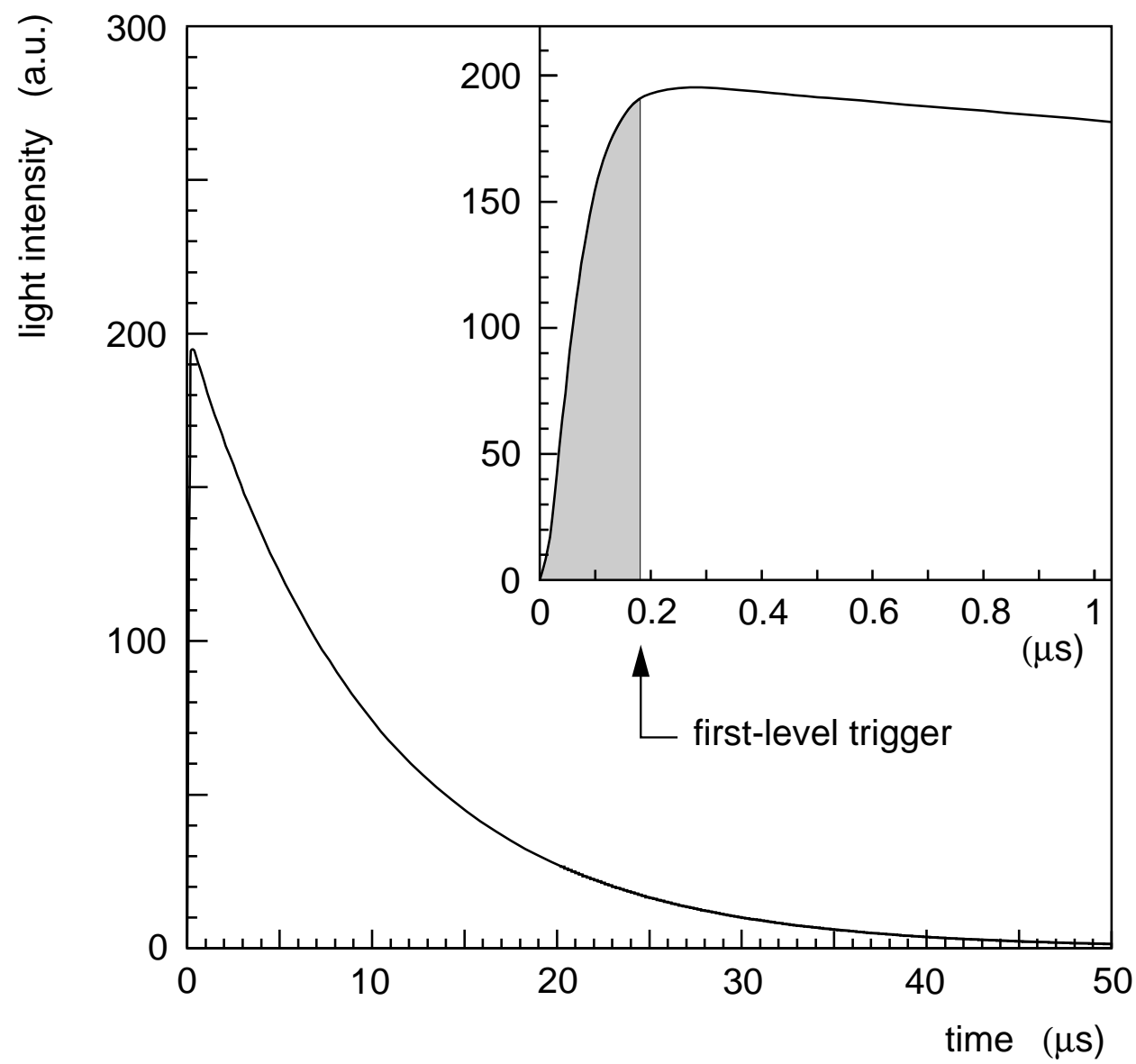

Figure 13: Monte-Carlo calculation of the variation with time of the light emitted by the image intensifier placed in front of the MCP tube (readout chain of the square bundle). The first microsecond has been expanded in the insert. The phosphors of the first three image intensifiers act as an optical memory, and only the light emitted before the implementation of the first-level trigger is lost (shaded area). 

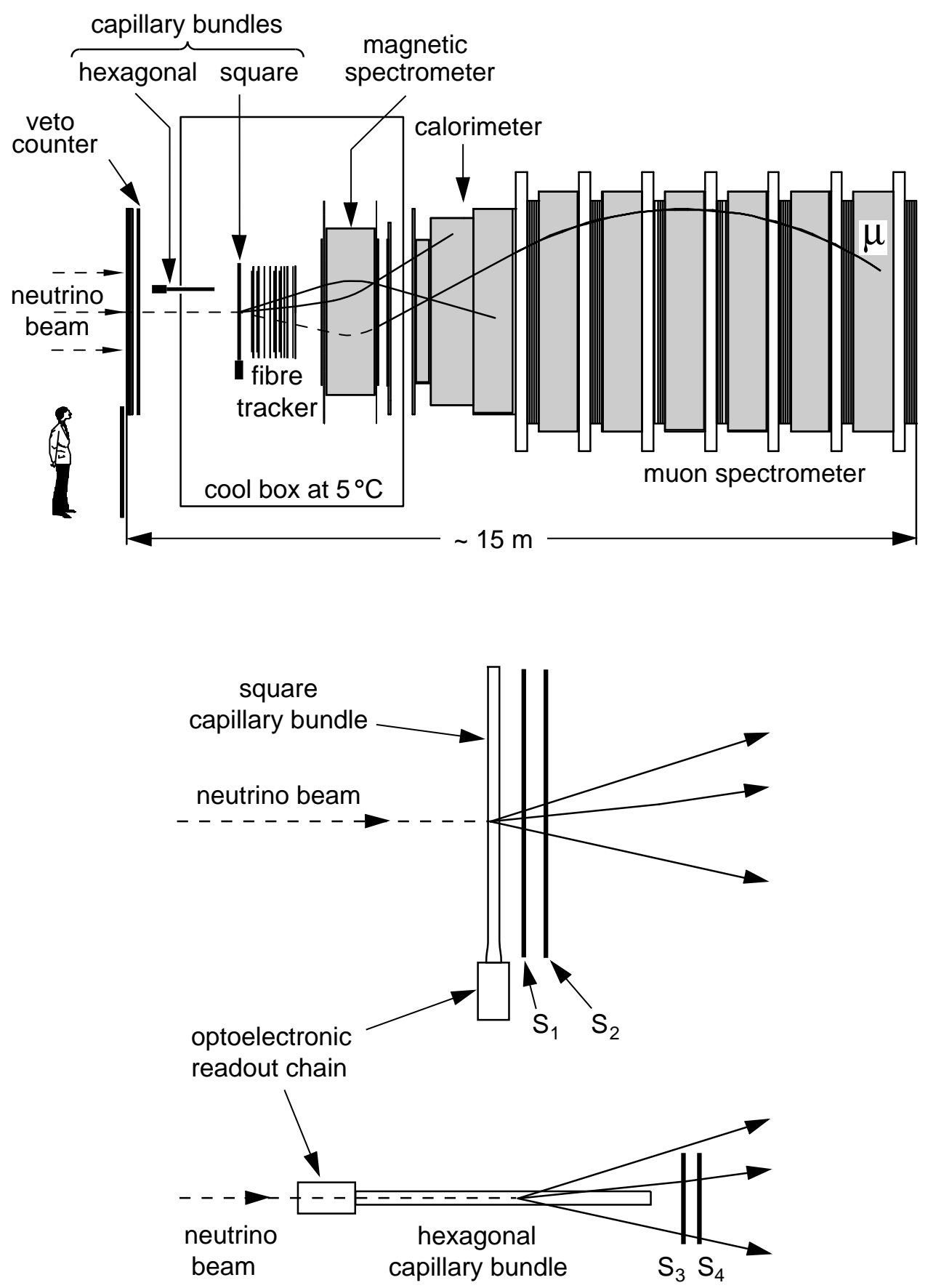

Figure 14: Setup of the capillary bundles in the CHORUS detector, for studies of neutrino interactions. The trigger-counter positions with respect to the capillary bundles are shown in the lower part of the figure, for square and hexagonal bundles. 

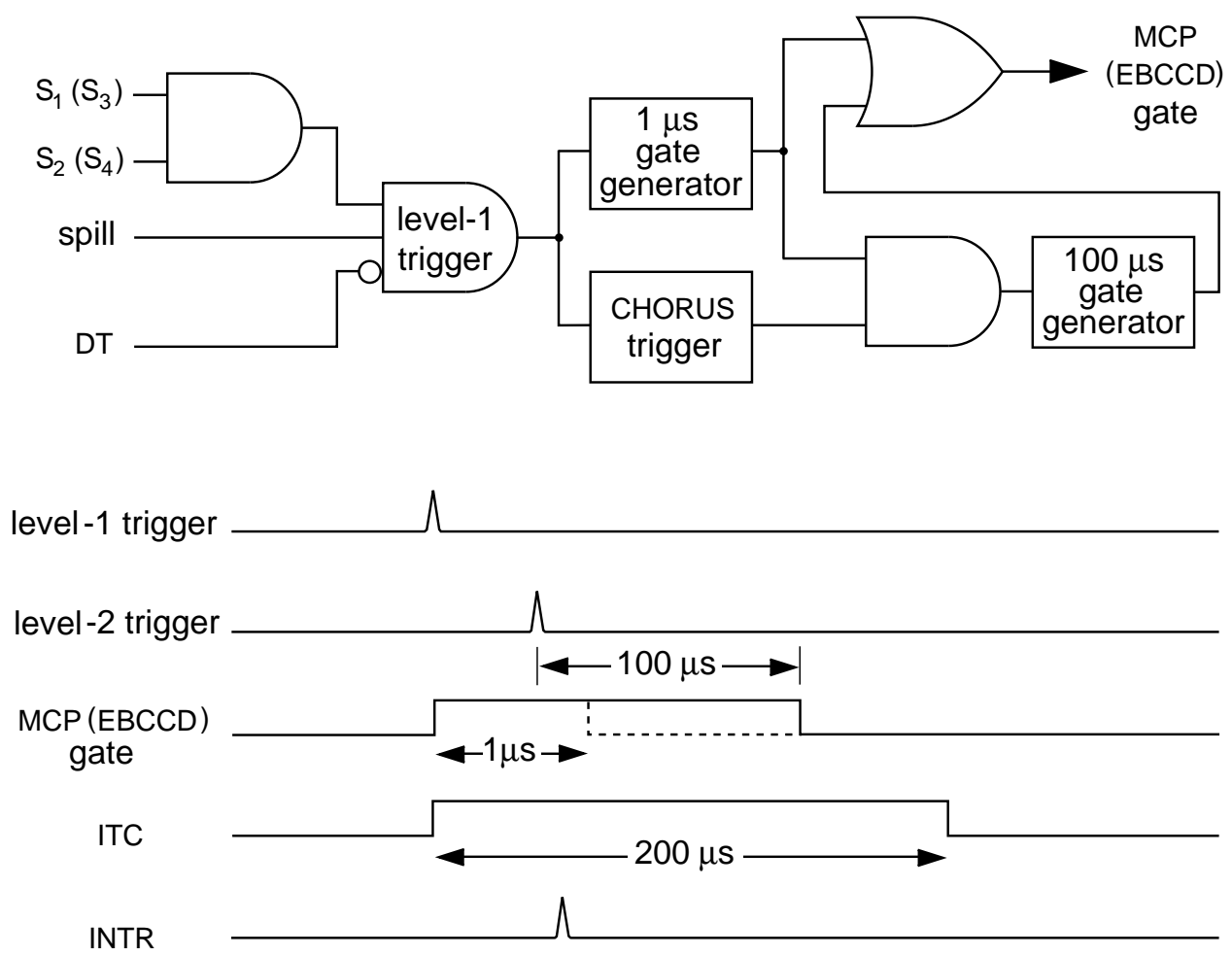

Figure 15: Scheme of the trigger logic for gating the MCP (EBCCD) in the readout chain of the square (hexagonal) bundle. The scintillator counters $S_{1}$ and $S_{2}\left(S_{3}\right.$ and $\left.S_{4}\right)$ are in coincidence with the beam spill and are in anticoincidence with a dead time (DT) pulse during the acquisition time of the CCD (EBCCD). The CCD chips were continuously cleared except during the integration-time control (ITC) pulse. The interrupt (INTR) starts the data acquisition.
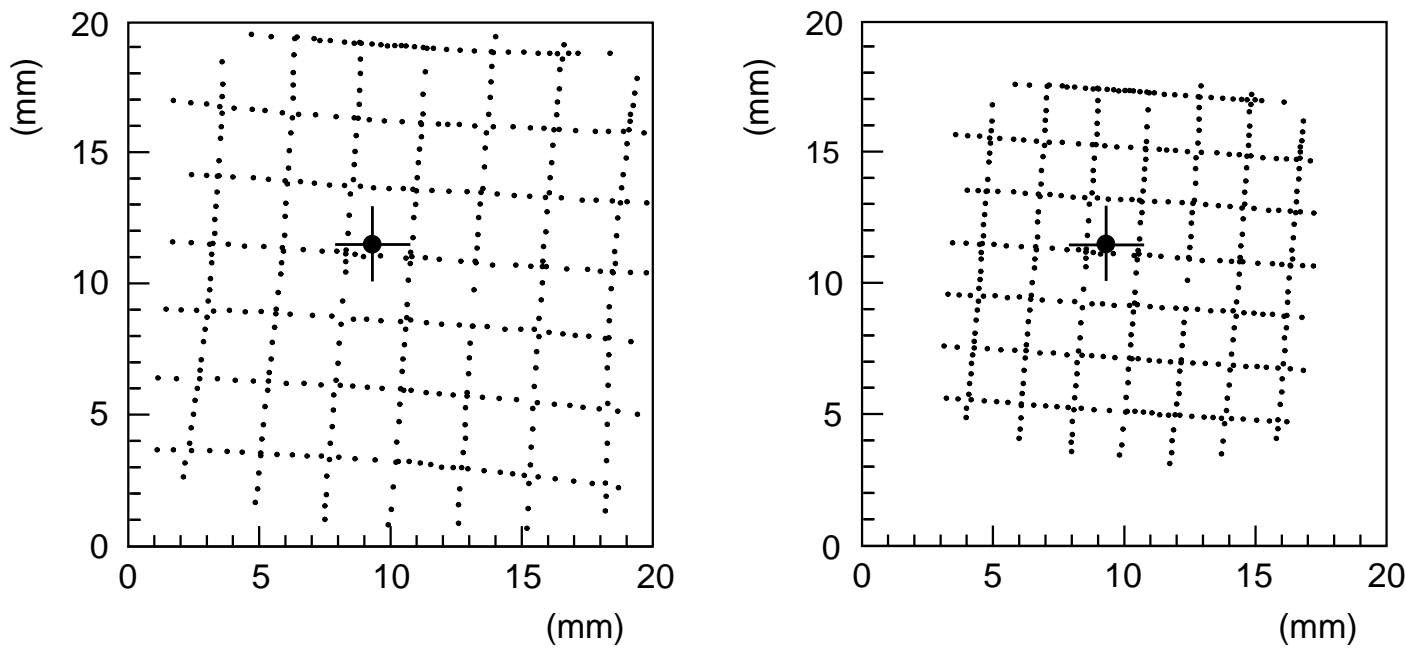

Figure 16: Pincushion distortion in the readout chain of the square bundle. On the left: image of the reference grid, as seen at the CCD. On the right: outline of the grid reconstructed in the real space of the bundle, after application of the corrections described in the text. The crosses indicate the position of the chain's optical centre. 

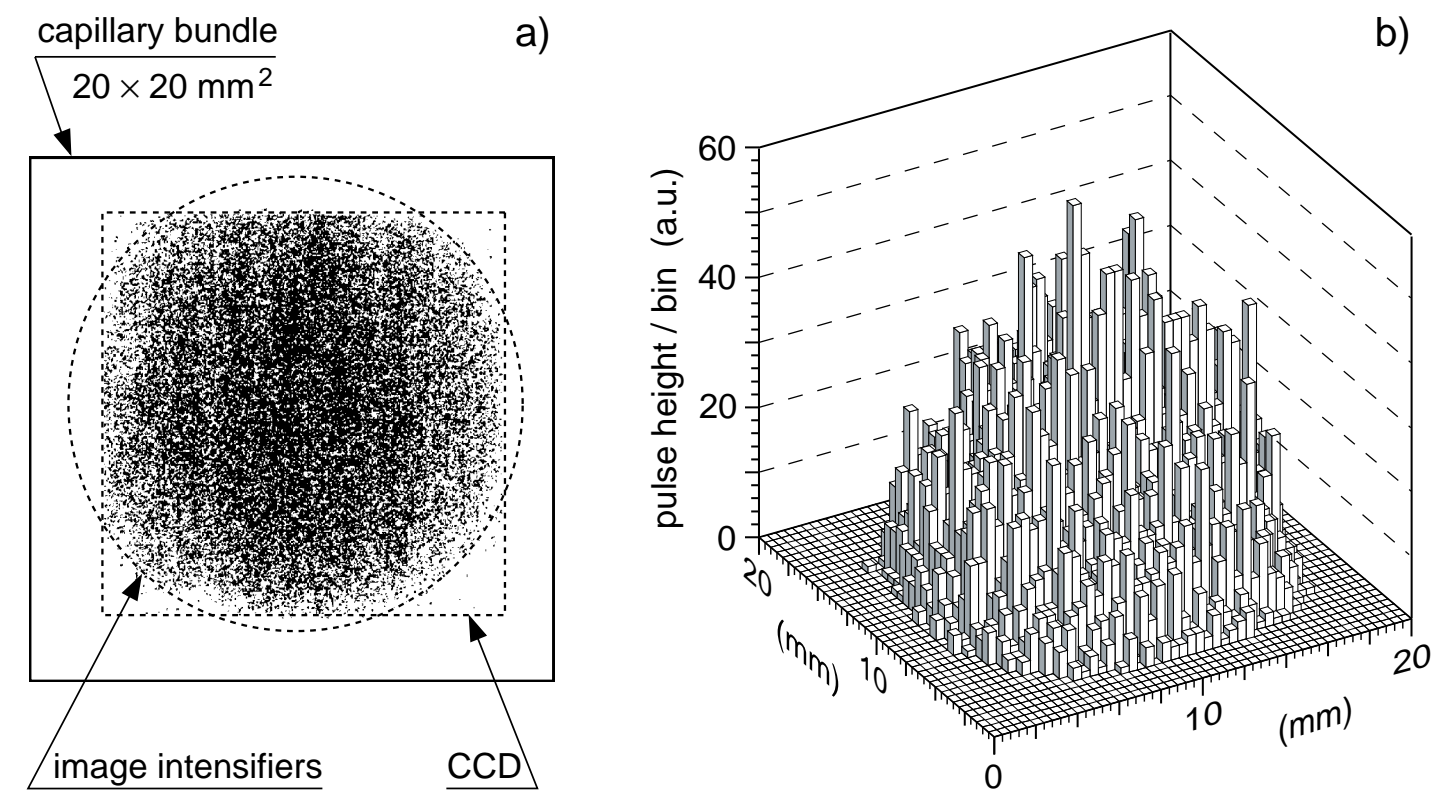

Figure 17: a) Distribution, in plane perpendicular to square bundle, of hits associated with muon tracks uniformly crossing the bundle. The dashed circle and square delimit the zones seen by the image-intensifier chain and by the CCD respectively. b) Same as in a) but with each hit weighted by its pulse height measured by the CCD. Near the edge of the accepted zone, vignetting causes a) a lower hit density and b) reduced signal light.

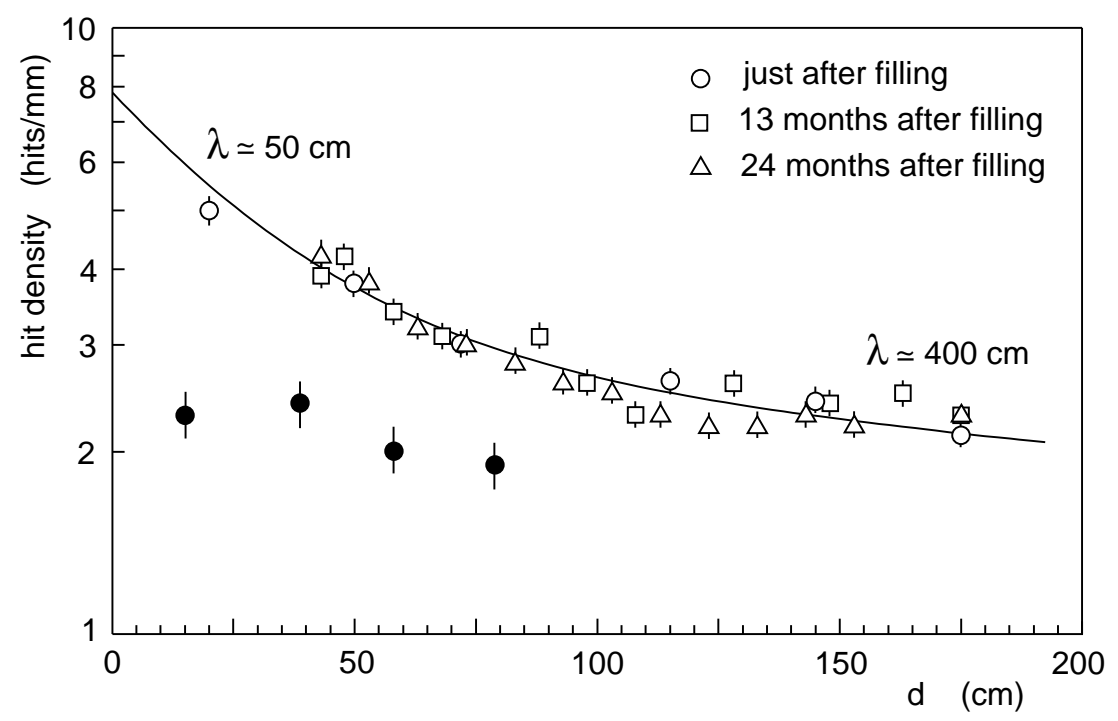

Figure 18: Hit densities measured along the tracks of minimum-ionizing particles crossing the bundles perpendicularly to their axes, as a function of the distance between the tracks and the readout end of the bundles. Open points relate to the square bundle and were taken at different times after the bundle filling. The curve represents the result of a fit of two exponentials to all the open points. Attenuation lengths near the square bundle's ends are indicated. Closed circles refer to the hexagonal bundle. 


\section{Results with square bundle}
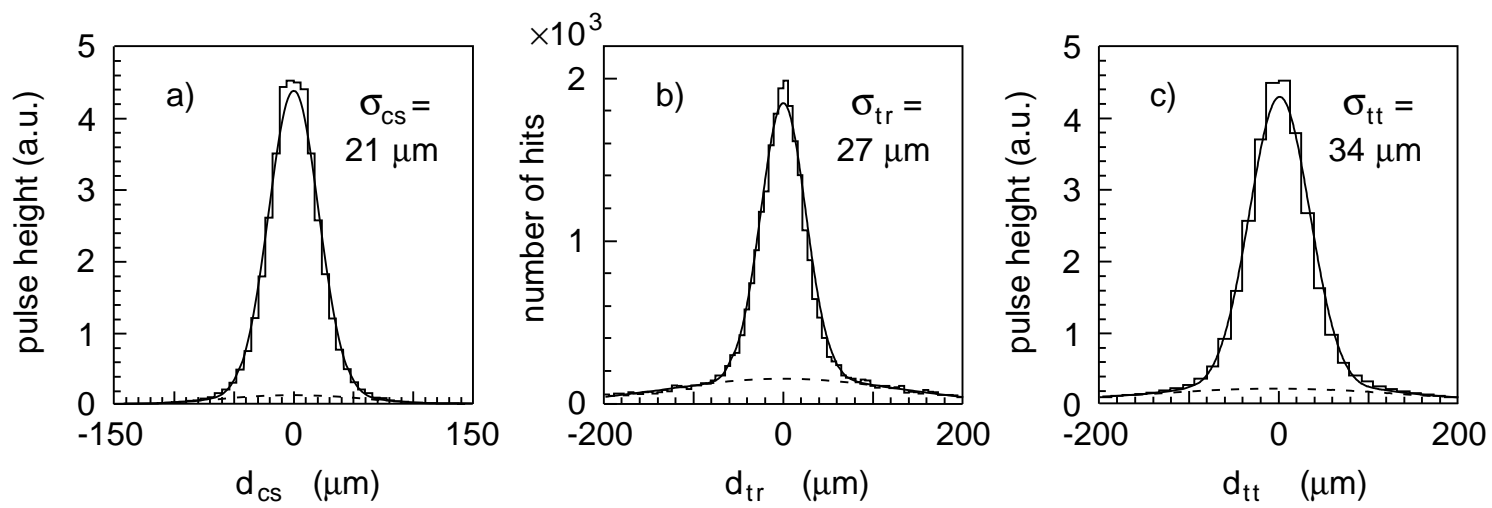

Results with hexagonal bundle
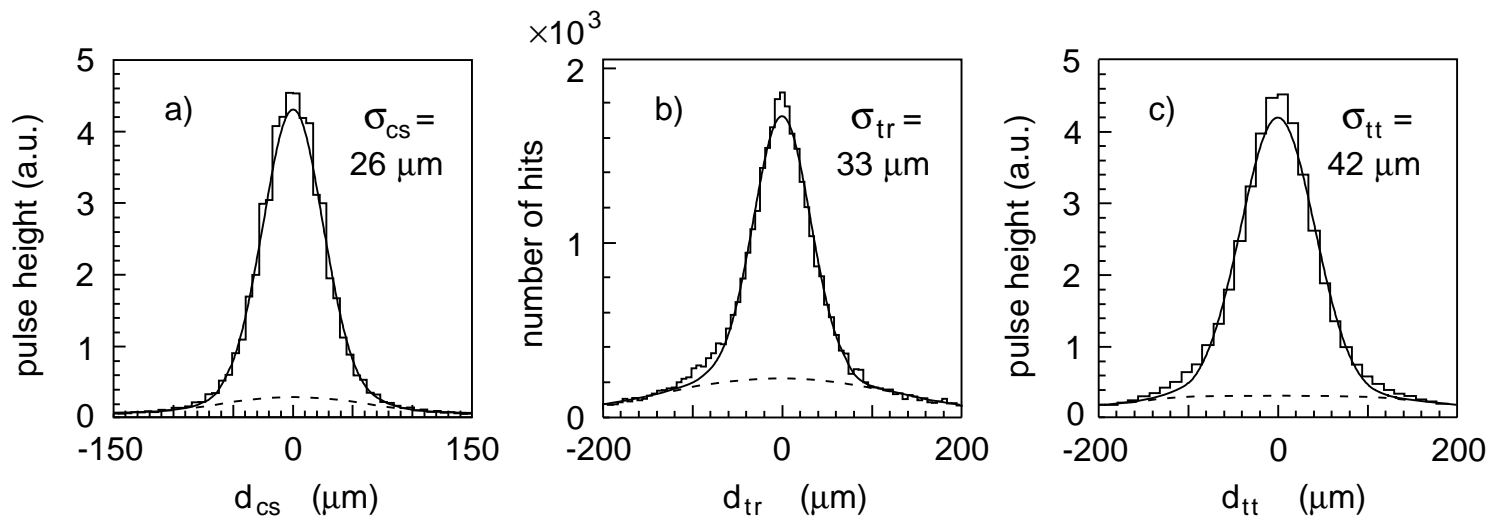

Figure 19: Distributions showing: a) variation of pulse height with projected distance $d_{c s}$ from a cluster's centroid; b) variation of number of hits with distance $d_{t r}$ from fitted track; c) variation of pulse height with distance $d_{t t}$ from fitted track. The upper and lower distributions refer respectively to the square bundle and to the hexagonal bundle. Each distribution is fitted by a Gaussian superimposed on a background. The standard deviations of the fitted Gaussians are reported. All distances are given in the real space of the capillary bundles. 

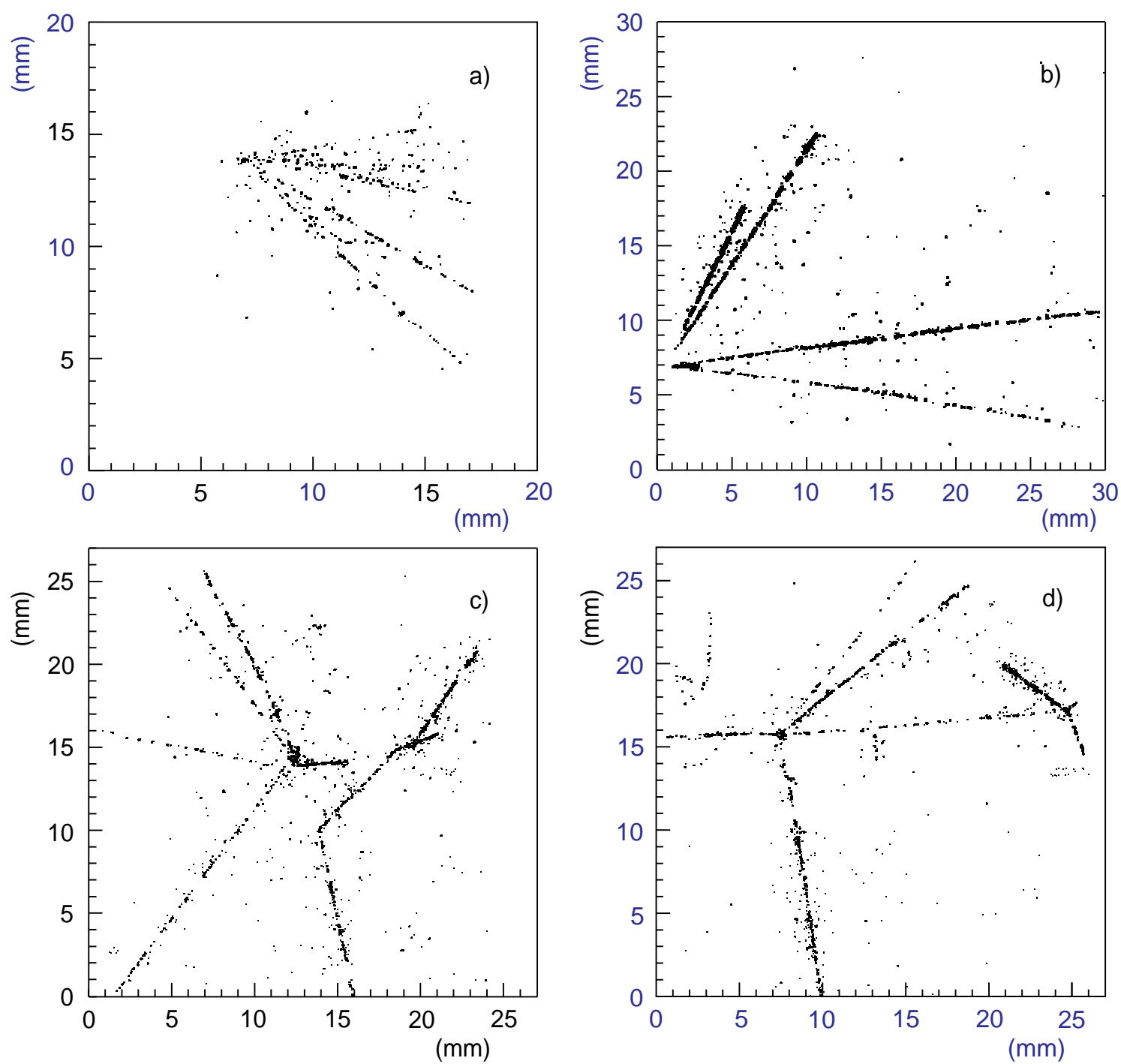

Figure 20: Images of neutrino interactions occurring: a), b) in the square bundle placed perpendicularly to the beam, neutrinos arriving from the left; c), d) in the hexagonal bundle placed longitudinally to the beam, neutrinos arriving perpendicularly to the figure. The interaction shown in b) takes place in the tapered part of the bundle, close to the readout window. The hit density observed along the tracks is consequently much higher than for the event shown in a). In the longitudinal configuration, the angle between the emitted particles and the capillary bundle is, on average, lower than in the transverse configuration. This is the reason for the enhanced hit densities observed in c) and $d$ ). 

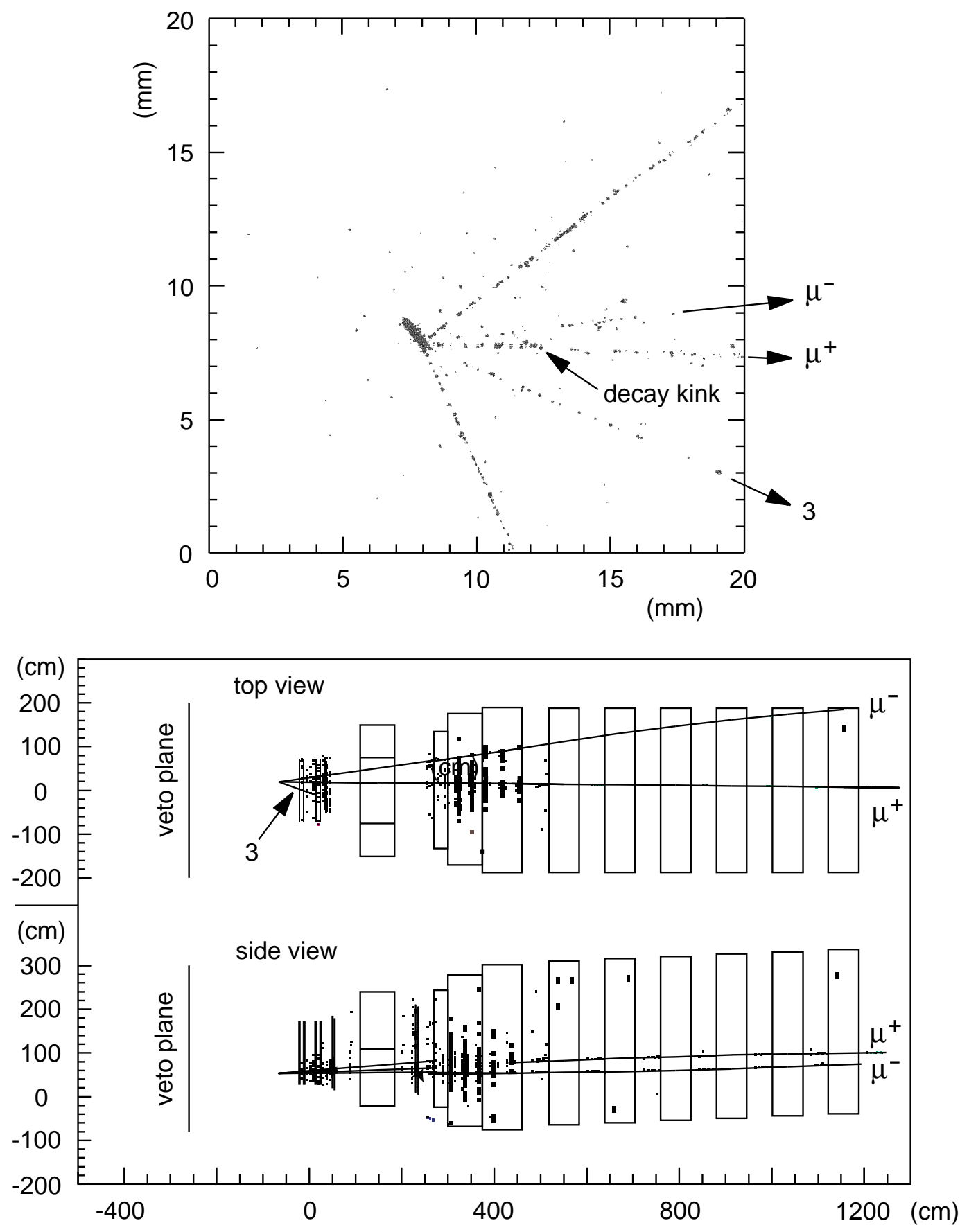

Figure 21: Neutrino interaction observed in the square capillary bundle and reconstructed in CHORUS. The neutrino arrives from the left. A decay vertex is identified through a small kink, confirmed by a change in the hit density (see text). A $\mu^{+}$, a $\mu^{-}$and a third charged particle (labelled " 3 ") are detected in the CHORUS apparatus. 